\title{
Using Identification and Quantification to Decipher The Dynamic Changes of Main Chemical Components In The Processing of Stir-Frying Codonopsis Radix With Rice By HPLC-MS/MS
}

\section{Qinghao Wang}

Henan University of Traditional Chinese Medicine

\section{Yinghao Zheng}

China Academy of Chinese Medical Sciences Institute of Chinese Materia Medica

\section{Yun Wang}

China Academy of Chinese Medical Sciences Institute of Chinese Materia Medica

\section{Dingrong Yu}

China Academy of Chinese Medical Sciences Institute of Chinese Materia Medica

\section{Zhenhong Lei}

Shanxi Zhendong Genuine Medicinal Materials Development Co., Ltd.

\section{Yulong Wang}

Shanxi Zhendong Genuine Medicinal Materials Development Co., Ltd.

\section{Tianliang Liu}

Beijing Tongrentang Co., Ltd.

Cun Zhang ( $\nabla$ zhc95@163.com )

Institute of Chinese Materia Medica, China Academy of Chinese Medical Sciences https://orcid.org/0000-0001-87387698

\section{Research}

Keywords: Rice-processed Codonopsis Radix, Chemical component, Dynamic changes, HPLC-MS/MS

Posted Date: December 1st, 2020

DOl: https://doi.org/10.21203/rs.3.rs-113817/v1

License: (a) (1) This work is licensed under a Creative Commons Attribution 4.0 International License. Read Full License 


\section{Abstract}

Background: Codonopsis Radix (CR) as the tonic Chinese herbis is good at benefiting Qi and promoting saliva, while riceprocessed CR (RCR) can alleviate the original dryness and more specialize in invigorating the spleen and stopping diarrhea. Despite CR and RCR are long-standing medicines commonly used in clinic, information on their differences in substance basis is limited, let alone dynamic changes of their main chemical components. Given this, a more comprehensive understanding of processing principles in CR is needed.

Methods: In this study, a strategy for rapid separation and identification of chemical constituents in crude CR and RCR was developed by HPLC-Q-TOF-MS/MS. Moreover, chemical constituents (lobetyolin and atractylenolide III) with significant content change were quantitatively determined by HPLC-QQQ-MS, 5-hydroxymethylfurfural (5-HMF) was quantified by HPLC, and codonopsis pilosula polysaccharides (CPPS) was measured by phenol-sulphuric acid method.

Results: A total of 28 compounds were identified from CR and RCR, including 12 organic acids, 6 phenylpropanoids, 5 hexanol glycosides and hexylene glycosides, 2 polyacetylenes, 1 alcohol compound, 1 sesquiterpene and 1 aldehyde. In order to explore the dynamic changes from raw $\mathrm{CR}$ to processed RCR, the content of representative components in several samples collected during processing was determined. The quantitative results indicated that the contents of lobetyolin, atractylenolide III and CPPS were lower in RCR than these in CR, and 5-HMF was newly formed in processed products, which manifested chemical composition change before and after processing.

Conclusions: Processing principles and dynamic changes of CR were preliminarily elucidated. Lobetyolin, atractylenolide III, 5-HMF and CPPS were recommended as quality markers to distinguish CR from RCR. Since the established method is rapid, accurate and readily applicable, it will be helpful for quality control and identification of crude CR and RCR.

\section{Background}

Codonopsis Radix (CR, Danshen in Chinese) is derived from the dried root of Codonopsis pilosula (Franch.) Nannf., Codonopsis pilosula Nannf.var.modesta (Nannf.) L.T.Shen, and Codonopsis tangshen Oliv. according to pharmacopoeia in China Pharmacopoeia 2015 Volume 1. It is mainly distributed in Gansu, Shanxi, Hubei and Sichuan provinces of China [1]. $\mathrm{CR}$ has various biological activities, including antifatigue, antiaging and immunomodulatory [2]. CR can be regarded as a supplement Qi Chinese medicine for the treatment of fatigue, spleen deficient, immune system and inappetence in the clinical application [3]. Based on the similar pharmacological activities with Panax ginseng, CR is also prescribed as a substitute for Panax ginseng in therapy [4]. Traditional processing methods of CR are recorded in standardized documents, including rice-processing [1], honey-processing [5] and bran-processing [6]. According to processing principle, rice-processed CR (RCR) possesses the function of invigorating Qi and strengthening spleen, honey-processed product with a burnt fragrant odor is responsible for benefiting Qi and nourishing Yin, and CR stir-frying with bran can reinforce spleen and harmonize stomach [7]. Thus, it can be seen that after processing with different excipients, efficacy of CR has different emphasis. Despite the wide use of CR, there is very little information on the different processed of CR.

Chinese herb medicines are reported to exert their curative effects through multiple components on multiple targets [8], so it is necessary to consider their multiple active constituents to evaluate the quality of herbal products[2]. CR consists of complicated chemical components, like polysaccharides, glycosides, triterpenoids, sterols, flavones, volatile oils, alkaloids, phenylpropanoids, polyacetylenes and organic acids $[9,10]$. Many components have been reported to be major contributors to curative effects of CR [11]. Lobetyolin is responsible for anti-gastric ulcer activity and protecting gastric mucosal from ethanol induced injury, which is consistent with the traditional effect of invigorating Qi and tonifying spleen in CR [12]. Atractylenolide III, a sesquiterpene component, makes impact on anti-inflammatory, anti-cancer, enhancing immunity and protecting liver [13]. It is known that 5-hydroxymethylfurfural (5-HMF) has positive influence in the flavor formation is a browning product of Mailard reaction, which was commonly found in the heating processing of traditional Chinese medicine (TCM) [14]. 5-HMF could be produced in RCR and contribute to caramel-like flavor, probably because sugars and 
acidic components such as ferulic acid in CR provided substances bases involving in Maillard reaction [15]. Codonopsis pilosula polysaccharides (CPPS) existed widely in CR, and its content will change during heating process, which is in relation to processing mechanism of $\mathrm{CR}$. While progress on the analysis of chemical compounds of CR has been impressive [2,16], the changes of whole chemical compositions in CR after processing have not been well studied, especially for RCR that is record in Chinese Pharmacopeia. Therefore, the deeply research of the chemical components and processing mechanism of RCR should be necessary to explore.

High-performance liquid chromatography/tandem mass spectrometry (HPLC-MS/MS) offers comprehensive advantages of high speed [17], improved sensitivity [18], superior accuracy [19], and can provide the molecules range from polar sugars and non-aromatic organic acids [20] to various lipids [21]. As a dominant technique for quantity and screening of specific chemicals in food and medicine fields [22], HPLC-MS/MS has been widely used to investigate the comprehensive profiles of natural products in herbal plants [23, 24], achieving to analyze the chemical components of TCM successfully [25]. HPLCMS/MS exhibits high resolution, and presents massive amount of information on molecular formula and fragmentation ions, which is greatly valuable to the structural inference of unknown ingredients [26-28].

In this study, the method established with HPLC-MS/MS was used to comprehensively analyze the components and compare differences between CR and RCR. The fragmentation behavior of major chemical components in analytes was explored in negative mode. Four major components that were suggested as characteristic components of RCR, including lobetyolin, atractylenolide III, 5-HMF and CPPS, were quantitatively measured by HPLC-QQQ-MS/MS, HPLC and phenolsulphuric acid method. This study not only provides an effective method for the rapid identification of CR and rice fried $\mathrm{RCR}$, but also demonstrates main changed components of $\mathrm{CR}$ before and after rice processing, suggesting a certain experimental foundation for revealing the processing principles and dynamic changes of CR.

\section{Methods}

\subsection{Chemicals and samples}

Acetonitrile (HPLC grade) was purchased from Fisher scientific (Pittsburgh, PA, USA). Formic acid (HPLC grade) was offered by Dikma (Beijing, China). Purified water was bought from Wahaha Co., Ltd. (Hangzhou, China). Other reagents used for sample preparation were of analytical grade. Lobetyolin, atractylenolide III and 5-HMF were purchased from Chengdu Must Bio-Technology Co., Ltd. (Chengdou, China), and the purity of each compound was above $98.0 \%$. CR from Gansu province was supplied by Shanxi Zhendong Genuine Medicinal Materials Development Co., Ltd. (Shanxi, China).The material was identified as the dried root of Codonopsis pilosula (Franch.) Nannf. by Professor Cun Zhang of the Institute of Chinese Materia Medical, China Academy of Chinese Medical Sciences.

\subsection{Preparation of CR and RCR}

CR was prepared according to the procedure recorded in the China Pharmacopoeia 2015 Volume 1. It simply means that after removing impurities, CR was washed clean and cut into $3 \mu \mathrm{m}$ thick slices, followed by drying.

Stir-frying CR with rice was prepared in accordance with the operating standard recorded in the China Pharmacopoeia 2015 Volume 1. Briefly, $2 \mathrm{~kg}$ japonica rice was put into a heated pot at the temperature $270{ }^{\circ} \mathrm{C}$, stir-fried constantly until it generated white smoke, followed by adding $10 \mathrm{~kg}$ CR during the next $1 \mathrm{~min}$. Next, multiple samples were collected every 1 min or 0.5 min until the surface of material turned dark yellow with charred points occasionally, and the final product was completed at $8.5 \mathrm{~min}$. Take out the drugs, sieve away the rice, and finally get RCR. CR and its processing products were numbered as Table 1. 
Table 1

Sample numbers of processing products of $\mathrm{CR}$.

\begin{tabular}{|lll|}
\hline No. & Processing time (min) & Sample number \\
\hline 1 & 0 & CR \\
\hline 2 & 1.0 & CR-1 \\
3 & 2.0 & CR-2 \\
\hline 4 & 3.0 & CR-3 \\
\hline 5 & 4.0 & CR-4 \\
\hline 6 & 5.0 & CR-5 \\
\hline 7 & 5.5 & CR-6 \\
\hline 8 & 6.0 & CR-7 \\
\hline 9 & 6.5 & CR-8 \\
\hline 10 & 7.0 & CR-9 \\
\hline 11 & 7.5 & CR-10 \\
\hline 12 & 8.0 & CR-11 \\
\hline 13 & 8.5 & RCR \\
\hline
\end{tabular}

\subsection{Preparation of sample extraction}

$\mathrm{CR}$ and its rice-processed products were pulverized with a grinder, and then passed through a 60-mesh sieve, respectively. The samples of CR and RCR were weighed precisely $(1.0 \mathrm{~g})$ and added into a 50-mL conical flask containing $25 \mathrm{~mL}$ methanol. Total quality was recorded. After ultrasonicated for $30 \mathrm{~min}$, samples were placed to cool, and then current weight was supplemented to the original weight with methanol. The solution was filtered through a $0.22 \mu \mathrm{m}$ nylon membrane filter for LC-MS or HPLC analysis.

Polysaccharide from CR and multiple rice-processed products was prepared. Briefly, $0.2 \mathrm{~g}$ sample with $50 \mathrm{~mL} 80 \%$ ethanol was extracted by reflux in a water bath for $2 \mathrm{~h}$ to remove fat-soluble substance, followed by filtering extract solution to obtain a residue. The residue after washing was extracted under reflux with $60 \mathrm{~mL}$ water for $2 \mathrm{~h}$, and then was filtered to collect filtrate, which were repeated three times. The mixed filtrate, namely polysaccharide solution, was concentrated to $100 \mathrm{~mL}$, and then dilute sample solution 10 times for further analysis.

\subsection{Standard solution preparation}

The stock solutions of lobetyolin, atractylenolide III and 5-HMF were prepared with concentration of $1.0 \mathrm{mg} / \mathrm{mL}$. Next, the stock solutions of lobetyolin and atractylenolide III were mixed, followed by serially diluting with methanol to obtain a series of working solutions of mixed standard solution. Likewise, series of working solutions of 5-HMF were prepared with methanol as diluent. Glucose used as reference substance for quantification of CPPS was serially diluted to form working solutions with different concentrations.

\subsection{HPLC-Q-TOF-MS analysis for identification of compositions}

Separation of components was implemented by Agilent HPLC system comprising an autosampler, thermostabilized column compartment and a binary pump. Chromatographic analysis was carried out with Diamonsil C18 column $(2.1 \mathrm{~mm} \times$ $150 \mathrm{~mm}, 3 \mu \mathrm{m})$ maintaining column temperature at $35^{\circ} \mathrm{C}$. The mobile phase was a binary eluent of $0.1 \%(\mathrm{v} / \mathrm{v})$ formic acid $(A)$ and acetonitrile $(B)$ with flowrate at $0.2 \mathrm{~mL} / \mathrm{min}$ using gradient conditions as follows: $0-5 \mathrm{~min}, 5 \% \mathrm{~B} ; 5-13 \mathrm{~min}, 5-13 \%$ 
B; 13-25 min, 13-23\% B; 25-35 min, 23-35\% B; 35-39 min, 35-50\% B; 39-47 min,50-65\% B; 47-67 min, 65-100\% B; 67-75 $\mathrm{min}, 100 \% \mathrm{~B}$. The injection volume was $10 \mu \mathrm{l}$, and detection wavelength was set as $268 \mathrm{~nm}[16]$.

The MS was conducted on an Agilent 6545 UHD Accurate-Mass Q-TOF/MS system with electrospray ionization interface. Temperature of sheath gas was set at $300{ }^{\circ} \mathrm{C}$ with a flow rate of $8 \mathrm{~L} / \mathrm{min}$. Nitrogen was used as the desolvation gas and delivered at $350{ }^{\circ} \mathrm{C}$ with gas flow of $8 \mathrm{~L} / \mathrm{min}$. Capillary voltage was $4.5 \mathrm{kV}$, nebulizer was $40 \mathrm{psi}$, and fragmentor voltage was set to $175 \mathrm{~V}$. Scan was performed with a mass/change $(\mathrm{m} / \mathrm{z})$ range of 100-1700 in negative mode.

Acquisition data from HPLC-MS/MS was analyzed using software of Agilent Qualitative Analysis 6.0 (Agilent Technologies, Santa Clara, CA, USA).

\subsection{HPLC-QQQ-MS analysis for quantification of lobetyolin and atractylenolide III}

An Agilent HPLC system coupled with an Agilent 6400 Triple Quadrupole Mass Spectrometer was employed for quantitative determination of lobetyolin and atractylenolide III. The HPLC conditions were identical with qualitative conditions, involving separation column, column temperature, sample volume, flow rate and mobile phase. The elution gradient was set as below: 0-5 min, 5-28\% B; 5-7 min, 28\% B; 7-10 min, 28-31\% B; 10-15 min, 31-80\% B; 15-19 min,80\% B.

The mass parameters were optimized using the standards and set in positive ion mode as follows: dry gas flow rate was $8 \mathrm{~L} / \mathrm{min}$ with the temperature at $350^{\circ} \mathrm{C}$; sheath gas temperature and flow rate were set to $250{ }^{\circ} \mathrm{C}$ and $8 \mathrm{~L} / \mathrm{min}$; capillary voltage was $3.5 \mathrm{kV}$; nebulizer was $45 \mathrm{psi}$. The fragmentor voltage and collision energy of lobetyolin were set at $200 \mathrm{~V}$ and $23 \mathrm{eV}$, while the fragmentor voltage and collision energy of atractylenolide III were set at $70 \mathrm{~V}$ and $5 \mathrm{eV}$. Positive ion mode was performed for quantitative analysis using the precursor to product ion combinations of $\mathrm{m} / \mathrm{z} 419.1 \rightarrow 256.7$ to detect lobetyolin and $\mathrm{m} / \mathrm{z} 249.1 \rightarrow 231.1$ and $249.1 \rightarrow 231.1$ for atractylenolide III.

\subsection{Method validation for HPLC-QQQ-MS}

Calibration curves were constructed by six concentration of lobetyolin and atractylenolide III covering the analytes levels expected in the tested samples, and linear ranges, regressive linear equations and correlation coefficients were determined. The precision assay was performed by examining intra-day and inter-day variations. The intra-day precision was assessed by repeated analyses for 6 times consecutively of RCR under optimized conditions, while inter-day precision was determined by analyzing the same sample solution on three consecutive days. The repeatability study was validated by investigating the same batch of six samples from RCR. Stability was reflected by assaying the sample of RCR at $0,2,4,6$, 12 and $24 \mathrm{~h}$. In the recovery test, the standard addition method was used to estimate the accuracy of the established method. Lobetyolin and atractylenolide III were added to the samples of RCR at the level equivalent to $100 \%$ of the known amount. The spiked samples were then extracted and analyzed with the optimized method. The recovery was evaluated by the following formula: [detected amount $(\mathrm{mg})$ - original amount $(\mathrm{mg})$ ] $/$ spiked amount $(\mathrm{mg}) \times 100 \%$. These results were presented via calculating relative standard deviation (RSD).

\subsection{HPLC analysis for quantification of 5-HMF}

A Shimadzu SIL-20A HPLC system equipped with a Diamonsil plus C18-column $(4.6 \mathrm{~mm} \times 250 \mathrm{~mm}, 5 \mu \mathrm{m})$ was applied for quantitative analysis of 5-HMF. The analysis method of samples was implemented as previously reported [29]. In brief, $10 \mu \mathrm{l}$ sample was analysed with column temperature at $30^{\circ} \mathrm{C}$. Flow rate was $0.8 \mathrm{~mL} / \mathrm{min}$ with mobile phase of $0.1 \%$ formic acid: acetonitrile (95:5) during $10 \mathrm{~min}$ of elution time. The wavelength was detected at $283 \mathrm{~nm}$. Finally, the standard curve of 5-HMF was established.

\subsection{Quantification of CPPS by phenol-sulphuric acid method}

Glucose used as a reference substance for quantification of CPPS was measured using phenol-sulfuric acid method. Briefly, $2 \mathrm{~mL}$ glucose solution was mixed with $1 \mathrm{~mL} 5 \%$ phenol, followed by $5 \mathrm{~mL}$ concentrated sulfuric acid. The mixture was 
reacted by heating in water bath for $15 \mathrm{~min}$ and then naturally cooled to room temperature. Distilled water was used as a blank for ultraviolet analysis, and glucose solution was measured at $490 \mathrm{~nm}$. CPPS was determined in accordance with above method.

\section{Results}

\subsection{Identification of major chemical components}

In preliminary experiment, the samples were comprehensively analyzed in both positive and negative ion mode under the optimized conditions. It was found that the sensitivity in negative ion mode was higher than in positive ion mode, and thus negative ion mode was applied to obtain fragmentation information of compounds in the study to capture base peak ion chromatograms (Fig. 1). Molecular formula of chemical constituents was accurately characterized in combination with MS analysis, CR database built in-home as well as related literature. In total, 28 compounds were identified from CR and RCR, which were considered as 7 types according to carbon skeletons, including 12 organic acids, 6 phenylpropanoids, 5 hexanol glycosides and hexylene glycosides, 2 polyacetylenes, 1 alcohol compound, 1 sesquiterpene and 1 aldehyde (Table 2). 
Table 2

Components identified in $\mathrm{CR}$ and RCR

\begin{tabular}{|c|c|c|c|c|c|c|c|c|c|c|}
\hline \multirow[t]{2}{*}{ No. } & \multirow{2}{*}{$\begin{array}{l}\text { RT } \\
(\min )\end{array}$} & \multirow[t]{2}{*}{ Formula } & \multirow[t]{2}{*}{ Precursor ion } & \multirow[t]{2}{*}{ ppm } & \multirow{2}{*}{$\begin{array}{l}\text { MS }^{2} \\
\text { fragment } \\
\text { ions in } \\
\text { negative } \\
\text { mode }\end{array}$} & \multirow{2}{*}{$\begin{array}{l}\text { Compound } \\
\text { name }\end{array}$} & \multirow[t]{2}{*}{ Type } & \multicolumn{2}{|c|}{ Source } & \multirow{2}{*}{$\begin{array}{l}\text { Peak } \\
\text { area } \\
\text { ratio } \\
\text { (CR: } \\
\text { RCR) }\end{array}$} \\
\hline & & & & & & & & CR & RCR & \\
\hline 1 & 3.15 & $\mathrm{C}_{4} \mathrm{H}_{6} \mathrm{O}_{4}$ & $\begin{array}{l}117.0192[\mathrm{M}- \\
\mathrm{H}]^{-}\end{array}$ & 1.60 & - & Succinic acid & a & + & + & 0.88 \\
\hline 2 & 5.10 & $\mathrm{C}_{6} \mathrm{H}_{6} \mathrm{O}_{3}$ & $\begin{array}{l}125.0251[\mathrm{M}- \\
\mathrm{H}]^{-}\end{array}$ & -7.24 & - & 5- HMF * & $b$ & - & + & - \\
\hline 3 & 12.48 & $\mathrm{C}_{7} \mathrm{H}_{6} \mathrm{O}_{3}$ & $\begin{array}{l}137.0240[\mathrm{M}- \\
\mathrm{H}]^{-}\end{array}$ & 2.10 & $\begin{array}{l}119.0261[\mathrm{M}- \\
\left.\mathrm{H}-\mathrm{H}_{2} \mathrm{O}\right]^{-}\end{array}$ & $\begin{array}{l}\text { 4- } \\
\text { Hydroxybenzoic } \\
\text { acid }\end{array}$ & a & + & + & 1.11 \\
\hline \multirow[t]{2}{*}{4} & \multirow[t]{2}{*}{14.51} & \multirow[t]{2}{*}{$\mathrm{C}_{17} \mathrm{H}_{24} \mathrm{O}_{9}$} & \multirow[t]{2}{*}{$\begin{array}{l}\text { 417.1406[M } \\
+\mathrm{HCOO}^{-}\end{array}$} & \multirow[t]{2}{*}{0.80} & $\begin{array}{l}371.1281[\mathrm{M}- \\
\mathrm{H}]^{-}\end{array}$ & \multirow[t]{2}{*}{ Syringin } & \multirow[t]{2}{*}{ c } & \multirow[t]{2}{*}{+} & \multirow[t]{2}{*}{+} & \multirow[t]{2}{*}{1.18} \\
\hline & & & & & $\begin{array}{l}210.0767[\mathrm{M}- \\
\left.\mathrm{H}-\mathrm{C}_{6} \mathrm{H}_{10} \mathrm{O}_{5}\right]^{-}\end{array}$ & & & & & \\
\hline 5 & 16.65 & $\mathrm{C}_{18} \mathrm{H}_{32} \mathrm{O}_{11}$ & $\begin{array}{l}423.1865[\mathrm{M}- \\
\mathrm{H}]^{-}\end{array}$ & 1.84 & $\begin{array}{l}261.0986[\mathrm{M}- \\
\left.\mathrm{H}-\mathrm{C}_{6} \mathrm{H}_{10} \mathrm{O}_{5}\right]^{-}\end{array}$ & $\begin{array}{l}\text { Trans-2-hexenyl- } \\
\beta \text {-glucoside }\end{array}$ & $d$ & + & + & 0.78 \\
\hline 6 & 19.69 & $\mathrm{C}_{18} \mathrm{H}_{32} \mathrm{O}_{11}$ & $\begin{array}{l}423.1867[\mathrm{M}- \\
\mathrm{H}]^{-}\end{array}$ & 1.05 & $\begin{array}{l}261.0985[\mathrm{M}- \\
\left.\mathrm{H}-\mathrm{C}_{6} \mathrm{H}_{10} \mathrm{O}_{5}\right]^{-}\end{array}$ & $\begin{array}{l}\text { Cis-2-hexenyl- } \beta \text { - } \\
\text { glucoside }\end{array}$ & $d$ & + & + & 1.25 \\
\hline \multirow[t]{3}{*}{7} & \multirow[t]{3}{*}{20.05} & \multirow[t]{3}{*}{$\mathrm{C}_{29} \mathrm{H}_{42} \mathrm{O}_{18}$} & \multirow[t]{3}{*}{$\begin{array}{l}677.2288[\mathrm{M}- \\
\mathrm{H}]^{-}\end{array}$} & 1.34 & $\begin{array}{l}497.1661[\mathrm{M}- \\
\left.\mathrm{H}-\mathrm{C}_{6} \mathrm{H}_{12} \mathrm{O}_{6}\right]^{-}\end{array}$ & \multirow[t]{3}{*}{ Tangshenoside I } & \multirow[t]{3}{*}{ c } & \multirow[t]{3}{*}{+} & \multirow[t]{3}{*}{+} & \multirow[t]{3}{*}{1.23} \\
\hline & & & & & $\begin{array}{l}453.1402[\mathrm{M}- \\
\mathrm{H}-\mathrm{C}_{6} \mathrm{H}_{12} \mathrm{O}_{6}^{-}\end{array}$ & & & & & \\
\hline & & & & & $\left.\mathrm{CO}_{2}\right]^{-}$ & & & & & \\
\hline \multirow[t]{3}{*}{8} & \multirow[t]{3}{*}{20.45} & \multirow[t]{3}{*}{$\mathrm{C}_{15} \mathrm{H}_{18} \mathrm{O}_{8}$} & \multirow[t]{3}{*}{$\begin{array}{l}325.0925[\mathrm{M}- \\
\mathrm{H}]^{-}\end{array}$} & 1.45 & $\begin{array}{l}163.0402[\mathrm{M}- \\
\left.\mathrm{H}-\mathrm{C}_{6} \mathrm{H}_{10} \mathrm{O}_{5}\right]^{-}\end{array}$ & \multirow{3}{*}{$\begin{array}{l}\text { 4-0-beta- } \\
\text { glucopyranosyl- } \\
\text { cis-coumaric } \\
\text { acid }\end{array}$} & \multirow[t]{3}{*}{ c } & + & + & 1.05 \\
\hline & & & & & $\begin{array}{l}119.0502[\mathrm{M}- \\
\mathrm{H}-\mathrm{C}_{6} \mathrm{H}_{10} \mathrm{O}_{5}^{-}\end{array}$ & & & & & \\
\hline & & & & & $\left.\mathrm{CO}_{2}\right]^{-}$ & & & & & \\
\hline 9 & 21.58 & $\mathrm{C}_{18} \mathrm{H}_{34} \mathrm{O}_{11}$ & $\begin{array}{l}471.2077[\mathrm{M} \\
+\mathrm{HCOO}^{-}\end{array}$ & 2.10 & $\begin{array}{l}425.2016[\mathrm{M}- \\
\mathrm{H}]^{-}\end{array}$ & $\begin{array}{l}\text { Hexy- } \beta \text {-a- } \\
\text { glucopyranosyl- } \\
(1 \rightarrow 2)-\beta-a-\end{array}$ & $d$ & + & + & 0.57 \\
\hline & & & & & $\begin{array}{l}263.1487[\mathrm{M}- \\
\left.\mathrm{H}-\mathrm{C}_{6} \mathrm{H}_{10} \mathrm{O}_{5}\right]^{-}\end{array}$ & glucopyranoside & & & & \\
\hline 10 & 22.43 & $\mathrm{C}_{18} \mathrm{H}_{34} \mathrm{O}_{11}$ & $\begin{array}{l}425.2026[\mathrm{M}- \\
\mathrm{H}]^{-}\end{array}$ & 1.33 & $\begin{array}{l}263.1438[\mathrm{M}- \\
\left.\mathrm{H}-\mathrm{C}_{6} \mathrm{H}_{10} \mathrm{O}_{5}\right]^{-}\end{array}$ & $\begin{array}{l}\text { Hexy- } \beta \text {-a- } \\
\text { glucopyranosyl- } \\
(1 \rightarrow 6)-\beta \text {-a- } \\
\text { glucopyranoside }\end{array}$ & $d$ & + & + & 0.61 \\
\hline
\end{tabular}

Type: a-organic acids; b-aldehyde compound; c-phenylpropanoids; d-hexanol glycosides and hexylene glycosides; epolyacetylenes; $f$-alcohol compound; g-sesquiterpene

* Identified as compound in standards. 


\begin{tabular}{|c|c|c|c|c|c|c|c|c|c|c|}
\hline \multirow[t]{2}{*}{ No. } & \multirow{2}{*}{$\begin{array}{l}\text { RT } \\
(\min )\end{array}$} & \multirow[t]{2}{*}{ Formula } & \multirow[t]{2}{*}{ Precursor ion } & \multirow[t]{2}{*}{ ppm } & \multirow{2}{*}{$\begin{array}{l}\text { MS }^{2} \\
\text { fragment } \\
\text { ions in } \\
\text { negative } \\
\text { mode }\end{array}$} & \multirow{2}{*}{$\begin{array}{l}\text { Compound } \\
\text { name }\end{array}$} & \multirow[t]{2}{*}{ Type } & \multicolumn{2}{|c|}{ Source } & \multirow{2}{*}{$\begin{array}{l}\text { Peak } \\
\text { area } \\
\text { ratio } \\
\text { (CR: } \\
\text { RCR) }\end{array}$} \\
\hline & & & & & & & & CR & RCR & \\
\hline \multirow[t]{3}{*}{11} & 23.42 & $\mathrm{C}_{21} \mathrm{H}_{26} \mathrm{O}_{12}$ & $\begin{array}{l}469.1351[\mathrm{M}- \\
\mathrm{H}]^{-}\end{array}$ & -0.21 & $\begin{array}{l}325.1046[\mathrm{M}- \\
\left.\mathrm{H}-\mathrm{C}_{6} \mathrm{H}_{8} \mathrm{O}_{4}\right]^{-}\end{array}$ & $\begin{array}{l}\text { Tangshenoside } \\
\text { V }\end{array}$ & c & + & + & 2.20 \\
\hline & & & & & $\begin{array}{l}163.0393[\mathrm{M}- \\
\mathrm{H}-\mathrm{C}_{6} \mathrm{H}_{8} \mathrm{O}_{4^{-}}\end{array}$ & & & & & \\
\hline & & & & & $\left.\mathrm{C}_{6} \mathrm{H}_{10} \mathrm{O}_{5}\right]^{-}$ & & & & & \\
\hline \multirow[t]{3}{*}{12} & 24.91 & $\mathrm{C}_{17} \mathrm{H}_{32} \mathrm{O}_{10}$ & $\begin{array}{l}395.1916[\mathrm{M}- \\
\mathrm{H}]^{-}\end{array}$ & 0.64 & $\begin{array}{l}263.1473[\mathrm{M}- \\
\left.\mathrm{H}-\mathrm{C}_{5} \mathrm{H}_{8} \mathrm{O}_{4}\right]^{-}\end{array}$ & $\begin{array}{l}\text { Pentose aldose } \\
\text { glucose-n- } \\
\text { hexanoside }\end{array}$ & $d$ & + & + & 1.00 \\
\hline & & & & & $\begin{array}{l}101.0355[\mathrm{M}- \\
\mathrm{H}-\mathrm{C}_{5} \mathrm{H}_{8} \mathrm{O}_{4^{-}}\end{array}$ & & & & & \\
\hline & & & & & $\left.\mathrm{C}_{6} \mathrm{H}_{10} \mathrm{O}_{5}\right]^{-}$ & & & & & \\
\hline \multirow[t]{3}{*}{13} & 25.31 & $\mathrm{C}_{26} \mathrm{H}_{38} \mathrm{O}_{13}$ & $\begin{array}{l}\text { 603.2292[M } \\
+\mathrm{HCOO}^{-}\end{array}$ & -0.52 & $\begin{array}{l}557.2239[\mathrm{M}- \\
\mathrm{H}]^{-}\end{array}$ & Lobetyolinin & $\mathrm{e}$ & + & + & 1.28 \\
\hline & & & & & $\begin{array}{l}467.1710[\mathrm{M}- \\
\left.\mathrm{H}-\mathrm{C}_{7} \mathrm{H}_{6}\right]^{-}\end{array}$ & & & & & \\
\hline & & & & & $\begin{array}{l}395.1934[\mathrm{M}- \\
\left.\mathrm{H}-\mathrm{C}_{6} \mathrm{H}_{10} \mathrm{O}_{5}\right]^{-}\end{array}$ & & & & & \\
\hline \multirow[t]{3}{*}{14} & 28.63 & $\mathrm{C}_{38} \mathrm{H}_{48} \mathrm{O}_{20}$ & $\begin{array}{l}823.2652[\mathrm{M}- \\
\mathrm{H}]^{-}\end{array}$ & 1.92 & $\begin{array}{l}497.1041[\mathrm{M}- \\
\mathrm{H}- \\
\left.\mathrm{C}_{15} \mathrm{H}_{18} \mathrm{O}_{8}\right]^{-}\end{array}$ & Codonoside B & c & + & + & 1.08 \\
\hline & & & & & $\begin{array}{l}452.9206[\mathrm{M}- \\
\mathrm{H}-\mathrm{C}_{15} \mathrm{H}_{18} \mathrm{O}_{8^{-}}\end{array}$ & & & & & \\
\hline & & & & & $\left.\mathrm{CO}_{2}\right]^{-}$ & & & & & \\
\hline \multirow[t]{2}{*}{15} & 28.87 & $\mathrm{C}_{20} \mathrm{H}_{28} \mathrm{O}_{8}$ & $\begin{array}{l}441.1780[\mathrm{M} \\
+\mathrm{HCOO}^{-}\end{array}$ & -4.48 & $\begin{array}{l}395.1922[\mathrm{M}- \\
\mathrm{H}]^{-}\end{array}$ & Lobetyolin * & e & + & + & 1.42 \\
\hline & & & & & $\begin{array}{l}215.1083[\mathrm{M}- \\
\left.\mathrm{H}-\mathrm{C}_{6} \mathrm{H}_{12} \mathrm{O}_{6}\right]^{-}\end{array}$ & & & & & \\
\hline \multirow[t]{3}{*}{16} & 30.40 & $\mathrm{C}_{38} \mathrm{H}_{48} \mathrm{O}_{20}$ & $823.264[\mathrm{M}-\mathrm{H}]$ & 2.52 & $\begin{array}{l}497.1040[\mathrm{M}- \\
\mathrm{H}- \\
\left.\mathrm{C}_{15} \mathrm{H}_{18} \mathrm{O}_{8}\right]^{-}\end{array}$ & Codonoside A & c & + & + & 2.08 \\
\hline & & & & & $\begin{array}{l}452.9196[\mathrm{M}- \\
\mathrm{H}-\mathrm{C}_{15} \mathrm{H}_{18} \mathrm{O}_{8^{-}}\end{array}$ & & & & & \\
\hline & & & & & $\left.\mathrm{CO}_{2}\right]^{-}$ & & & & & \\
\hline
\end{tabular}

Type: a-organic acids; b-aldehyde compound; c-phenylpropanoids; d-hexanol glycosides and hexylene glycosides; epolyacetylenes; f-alcohol compound; g-sesquiterpene

* Identified as compound in standards. 


\begin{tabular}{|c|c|c|c|c|c|c|c|c|c|c|}
\hline \multirow[t]{2}{*}{ No. } & \multirow{2}{*}{$\begin{array}{l}\text { RT } \\
(\min )\end{array}$} & \multirow[t]{2}{*}{ Formula } & \multirow[t]{2}{*}{ Precursor ion } & \multirow[t]{2}{*}{ ppm } & \multirow{2}{*}{$\begin{array}{l}\text { MS }^{2} \\
\text { fragment } \\
\text { ions in } \\
\text { negative } \\
\text { mode }\end{array}$} & \multirow{2}{*}{$\begin{array}{l}\text { Compound } \\
\text { name }\end{array}$} & \multirow[t]{2}{*}{ Type } & \multicolumn{2}{|c|}{ Source } & \multirow{2}{*}{$\begin{array}{l}\text { Peak } \\
\text { area } \\
\text { ratio } \\
\text { (CR: } \\
\text { RCR) }\end{array}$} \\
\hline & & & & & & & & CR & RCR & \\
\hline \multirow[t]{2}{*}{17} & 31.89 & $\mathrm{C}_{9} \mathrm{H}_{16} \mathrm{O}_{4}$ & $\begin{array}{l}187.0980[\mathrm{M}- \\
\mathrm{H}]^{-}\end{array}$ & 2.67 & $\begin{array}{l}169.0860[\mathrm{M}- \\
\left.\mathrm{H}-\mathrm{H}_{2} \mathrm{O}\right]^{-}\end{array}$ & Azelaic acid & a & + & + & 0.97 \\
\hline & & & & & $\begin{array}{l}125.0971[\mathrm{M}- \\
\left.\mathrm{H}-\mathrm{H}_{2} \mathrm{O}-\mathrm{CO}_{2}\right]^{-}\end{array}$ & & & & & \\
\hline \multirow[t]{3}{*}{18} & 37.52 & $\mathrm{C}_{18} \mathrm{H}_{32} \mathrm{O}_{5}$ & $\begin{array}{l}327.2176[\mathrm{M}- \\
\mathrm{H}]^{-}\end{array}$ & 0.32 & $\begin{array}{l}229.1405[\mathrm{M}- \\
\left.\mathrm{H}-\mathrm{C}_{6} \mathrm{H}_{10} \mathrm{O}\right]^{-}\end{array}$ & Malyngic acid & a & + & + & 1.56 \\
\hline & & & & & $\begin{array}{l}211.1351[\mathrm{M}- \\
\mathrm{H}-\mathrm{C}_{6} \mathrm{H}_{10} \mathrm{O}-\end{array}$ & & & & & \\
\hline & & & & & $\left.\mathrm{H}_{2} \mathrm{O}\right]^{-}$ & & & & & \\
\hline \multirow[t]{3}{*}{19} & 39.10 & $\mathrm{C}_{18} \mathrm{H}_{34} \mathrm{O}_{5}$ & $\begin{array}{l}329.2337[\mathrm{M}- \\
\mathrm{H}]^{-}\end{array}$ & -0.96 & $\begin{array}{l}229.1436[\mathrm{M}- \\
\left.\mathrm{H}-\mathrm{C}_{6} \mathrm{H}_{12} \mathrm{O}\right]^{-}\end{array}$ & Tianshi acid & a & + & + & 1.50 \\
\hline & & & & & $\begin{array}{l}211.1340[\mathrm{M}- \\
\mathrm{H}-\mathrm{C}_{6} \mathrm{H}_{12} \mathrm{O}-\end{array}$ & & & & & \\
\hline & & & & & $\left.\mathrm{H}_{2} \mathrm{O}\right]^{-}$ & & & & & \\
\hline 20 & 43.46 & $\mathrm{C}_{18} \mathrm{H}_{30} \mathrm{O}_{4}$ & $\begin{array}{l}309.2057[\mathrm{M}- \\
\mathrm{H}]^{-}\end{array}$ & 4.84 & $\begin{array}{l}291.1772[\mathrm{M}- \\
\left.\mathrm{H}-\mathrm{H}_{2} \mathrm{O}\right]^{-}\end{array}$ & $\begin{array}{l}\text { 6- } \\
\text { Methylgingediol }\end{array}$ & $f$ & + & + & 2.06 \\
\hline \multirow[t]{2}{*}{21} & 44.09 & $\mathrm{C}_{9} \mathrm{H}_{16} \mathrm{O}_{2}$ & $\begin{array}{l}311.2255[2 \mathrm{M}- \\
\mathrm{H}]^{-}\end{array}$ & -8.10 & $\begin{array}{l}\text { 155.1068[M- } \\
\mathrm{H}]^{-}\end{array}$ & 8-Nonenoic acid & a & + & + & 0.62 \\
\hline & & & & & $\begin{array}{l}127.1127[\mathrm{M}- \\
\left.\mathrm{H}-\mathrm{C}_{2} \mathrm{H}_{4}\right]^{-}\end{array}$ & & & & & \\
\hline 22 & 44.50 & $\mathrm{C}_{15} \mathrm{H}_{20} \mathrm{O}_{3}$ & $\begin{array}{l}247.1352[\mathrm{M}- \\
\mathrm{H}]^{-}\end{array}$ & 2.28 & $\begin{array}{l}203.1445[\mathrm{M}- \\
\left.\mathrm{H}-\mathrm{CO}_{2}\right]^{-}\end{array}$ & $\begin{array}{l}\text { Atractylenolide } \\
\text { III * }\end{array}$ & $g$ & + & + & 1.01 \\
\hline 23 & 46.30 & $\mathrm{C}_{18} \mathrm{H}_{34} \mathrm{O}_{4}$ & $\begin{array}{l}313.2397[\mathrm{M}- \\
\mathrm{H}]^{-}\end{array}$ & -4.07 & $\begin{array}{l}201.1137[\mathrm{M}- \\
\left.\mathrm{H}-\mathrm{C}_{8} \mathrm{H}_{16}\right]^{-}\end{array}$ & Leukotoxin diol & $\mathrm{a}$ & + & + & 0.73 \\
\hline 24 & 46.93 & $\mathrm{C}_{9} \mathrm{H}_{16} \mathrm{O}_{2}$ & $\begin{array}{l}311.2233[2 \mathrm{M}- \\
\mathrm{H}]^{-}\end{array}$ & -2.13 & $\begin{array}{l}\text { 155.1065[M- } \\
\mathrm{H}]^{-}\end{array}$ & 2-Nonenoic acid & a & + & + & 13.45 \\
\hline 25 & 51.07 & $\mathrm{C}_{18} \mathrm{H}_{32} \mathrm{O}_{3}$ & $\begin{array}{l}295.2287[\mathrm{M}- \\
\mathrm{H}]^{-}\end{array}$ & 1.31 & $\begin{array}{l}277.2159[\mathrm{M}- \\
\left.\mathrm{H}-\mathrm{H}_{2} \mathrm{O}\right]^{-}\end{array}$ & Coronaric acid & $\mathrm{a}$ & + & + & 3.12 \\
\hline 26 & 59.63 & $\mathrm{C}_{18} \mathrm{H}_{30} \mathrm{O}_{2}$ & $\begin{array}{l}277.2186[\mathrm{M}- \\
\mathrm{H}]^{-}\end{array}$ & -5.67 & $\begin{array}{l}233.1137[\mathrm{M}- \\
\left.\mathrm{H}-\mathrm{CO}_{2}\right]^{-}\end{array}$ & $\begin{array}{l}5,9,12- \\
\text { Octadecatrienoic } \\
\text { acid }\end{array}$ & a & + & + & 1.52 \\
\hline 27 & 62.78 & $\mathrm{C}_{18} \mathrm{H}_{32} \mathrm{O}_{2}$ & $\begin{array}{l}279.2334[\mathrm{M}- \\
\mathrm{H}]^{-}\end{array}$ & -1.65 & $\begin{array}{l}261.2221[\mathrm{M}- \\
\left.\mathrm{H}-\mathrm{H}_{2} \mathrm{O}\right]^{-}\end{array}$ & $\begin{array}{l}(9 Z, 12 Z)- \\
\text { Octadeca-9,12- } \\
\text { dienoic acid }\end{array}$ & a & + & + & 1.63 \\
\hline
\end{tabular}

Type: a-organic acids; b-aldehyde compound; c-phenylpropanoids; d-hexanol glycosides and hexylene glycosides; epolyacetylenes; f-alcohol compound; g-sesquiterpene

* Identified as compound in standards. 


\begin{tabular}{|c|c|c|c|c|c|c|c|c|c|c|}
\hline \multirow[t]{2}{*}{ No. } & \multirow{2}{*}{$\begin{array}{l}\text { RT } \\
(\min )\end{array}$} & \multirow[t]{2}{*}{ Formula } & \multirow[t]{2}{*}{ Precursor ion } & \multirow[t]{2}{*}{ ppm } & \multirow{2}{*}{$\begin{array}{l}\mathrm{MS}^{2} \\
\text { fragment } \\
\text { ions in } \\
\text { negative } \\
\text { mode }\end{array}$} & \multirow{2}{*}{$\begin{array}{l}\text { Compound } \\
\text { name }\end{array}$} & \multirow[t]{2}{*}{ Type } & \multicolumn{2}{|c|}{ Source } & \multirow{2}{*}{$\begin{array}{l}\text { Peak } \\
\text { area } \\
\text { ratio } \\
\text { (CR: } \\
\text { RCR) }\end{array}$} \\
\hline & & & & & & & & CR & RCR & \\
\hline 28 & 65.98 & $\mathrm{C}_{16} \mathrm{H}_{32} \mathrm{O}_{2}$ & $\begin{array}{l}255.2327[\mathrm{M}- \\
\mathrm{H}]^{-}\end{array}$ & 2.48 & $\begin{array}{l}237.2202[\mathrm{M}- \\
\left.\mathrm{H}-\mathrm{H}_{2} \mathrm{O}\right]^{-}\end{array}$ & Palmitic acid & a & + & + & 1.26 \\
\hline \multicolumn{11}{|c|}{$\begin{array}{l}\text { Type: a-organic acids; b-aldehyde compound; c-phenylpropanoids; d-hexanol glycosides and hexylene glycosides; e- } \\
\text { polyacetylenes; f-alcohol compound; g-sesquiterpene }\end{array}$} \\
\hline
\end{tabular}

Figure 1 Base peak ion chromatograms of CR (a) and RCR (b) in negative ionization mode.

Table 2 Components identified in CR and RCR

\subsubsection{Structural characterization and identification of aldehyde}

By comparison with mass data of literature, compound 2 was tentatively ascertained as 5-HMF [15], a crucial ingredient only in the processed product to differentiate $\mathrm{CR}$ from RCR, displaying a precursor the $[\mathrm{M}-\mathrm{H}]^{-}$ion at $m / z 125.0241\left(\mathrm{C}_{6} \mathrm{H}_{6} \mathrm{O}_{3}\right.$, mass error $=-7.24 \mathrm{ppm}$ ). The spectrum of MS analysis and molecular structure of compound 2 was shown in Fig. 2.

Figure 2 The mass spectra and the molecular structure of 5-HMF in negative ion mode. (a) mass spectra; (b) molecular structure.

\subsubsection{Structural characterization and identification of organic acids}

There were 12 constituents classified as organic acid from CR and RCR, and the trend of changed content of organic acid could be determined preliminarily by comparing peak area of these chemical components, presenting declines in 8 kinds of organic acids contents to varying degrees except for compounds 1, 17, 21 and 23. Generally, destruction and decomposition of organic acids would occur with heating-processed [30], thus making content decreased. Research has shown that excessive acidic compositions were liable to irritation, especially adverse effects on patients with weakness or ulcers [31]. Therefore, organic acid with a content decline in RCR shown correspondingly lower irritation, which might be related to supplementing deficiency and protecting spleen and stomach of RCR.

Compound 1 generated a quasi-molecular ion at $\mathrm{m} / \mathrm{z} 117.0192[\mathrm{M}-\mathrm{H}]^{-}$with molecular formula $\mathrm{C}_{4} \mathrm{H}_{6} \mathrm{O}_{4}$ (mass error = 1.60 ppm). Comparing with the literature data[16], compound 1 was tentatively identified as succinic acid.

Compound 3 showed $[\mathrm{M}-\mathrm{H}]^{-}$ion at $m / z 137.0240$, and was further fragmented into product ion at $m / z 119.0261$, resulting from the neutral loss of $\mathrm{H}_{2} \mathrm{O}$ [32]. In combination with the literature data [33], compound 3 was tentatively annotated as 4hydroxybenzoic acid.

Compound 17 exhibited its quasi-molecular ion at $m / z 187.0971[\mathrm{M}-\mathrm{H}]^{-}$with molecular formula $\mathrm{C}_{9} \mathrm{H}_{16} \mathrm{O}_{4}$. In addition, the precursor ion generated characteristic fragment ions at $m / z 169.0860\left[\mathrm{M}-\mathrm{H}-\mathrm{H}_{2} \mathrm{O}\right]^{-}$and $125.0969\left[\mathrm{M}-\mathrm{H}-\mathrm{H}_{2} \mathrm{O}-\mathrm{CO}_{2}\right]^{-}$in the $\mathrm{MS}^{2}$ spectrum, suggesting the neutral loss of $\mathrm{H}_{2} \mathrm{O}$ followed by losing $\mathrm{CO}_{2}$. Based on relevant literature [33], compound 17 was tentatively identified as azelaic acid, and its MS/MS spectra and fragmentation pathway were exhibited in Fig. 3.

Figure 3 The MS/MS spectra and the fragmentation pathways of azelaic acid in negative ion mode. (a) MS/MS spectra; (b) fragmentation pathways. 
Compound 18 produced a quasi-molecular ion at $m / z 327.2176[\mathrm{M}-\mathrm{H}]^{-}$with molecular formula $\mathrm{C}_{18} \mathrm{H}_{32} \mathrm{O}_{5}$. The deprotonated molecular ions further generated a series of ions at $m / z 229.1405\left[\mathrm{M}-\mathrm{H}-\mathrm{C}_{6} \mathrm{H}_{10} \mathrm{O}\right]^{-}$and $m / z 211.1351\left[\mathrm{M}-\mathrm{H}-\mathrm{C}_{6} \mathrm{H}_{10} \mathrm{O}-\mathrm{H}_{2} \mathrm{O}\right]^{-}$, which was tentatively identified as malyngic acid. Compound 19 with elemental composition of $\mathrm{C}_{18} \mathrm{H}_{34} \mathrm{O}_{5}$ shared the similar route to generate fragment ions as above. Combining with the reported data [34], compound 19 was tentatively ascertained as tianshi acid.

Compound 21 and 24 were a pair of isomers with identical molecular formula $\mathrm{C}_{9} \mathrm{H}_{16} \mathrm{O}_{2}$. Both of them exhibited [2M-H] ${ }^{-}$at $m / z 311.2255$, and deprotonated molecular ions at $m / z 155.1068[\mathrm{M}-\mathrm{H}]^{-}$in spectra, which were primarily identified 4nonenoic acid/ 8-nonenoic acid. The characteristic fragmentation of $\left[\mathrm{M}-\mathrm{H}-\mathrm{C}_{2} \mathrm{H}_{4}\right]^{-}$at $m / z 127.1127$ contributed to confirmation of 8-nonenoic acid due to the loss of $\mathrm{C}_{2} \mathrm{H}_{4}$. By combining with mass spectrometry information and related literature[16], compound 21 was tentatively identified to be 8-nonenoic acid, while compound 24 was annotated as 2nonenoic acid.

Compound 23 yielded molecular ion at $\mathrm{m} / \mathrm{z} 313.2397[\mathrm{M}-\mathrm{H}]^{-}$with molecular formula $\mathrm{C}_{18} \mathrm{H}_{34} \mathrm{O}_{4}$. The characteristic fragment ion at $\mathrm{m} / \mathrm{z} 201.1137\left[\mathrm{M}-\mathrm{H}-\mathrm{C}_{8} \mathrm{H}_{16}\right]^{-}$was observed in $\mathrm{MS}^{2}$ spectra. According to the information of literature[34], it was tentatively characterized as leukotoxin diol.

Compound 25 displayed precursor ion at $m / z 295.2277[\mathrm{M}-\mathrm{H}]^{-}$with molecular formula $\mathrm{C}_{18} \mathrm{H}_{32} \mathrm{O}_{3}$. It generated fragment ions at $m / z 277.2164\left[\mathrm{M}-\mathrm{H}_{-} \mathrm{H}_{2} \mathrm{O}\right]^{-}$, resulting from the neutral loss of $\mathrm{H}_{2} \mathrm{O}$. By comparison with references [33], compound 25 was identified as coronaric acid.

Compound 26 yielded its quasi-molecular ion at $m / z 277.2186[\mathrm{M}-\mathrm{H}]^{-}$with molecular formula $\mathrm{C}_{18} \mathrm{H}_{30} \mathrm{O}_{2}$. The $[\mathrm{M}-\mathrm{H}]^{-}$ion generated fragment ions at $\mathrm{m} / \mathrm{z} 233.1137\left[\mathrm{M}-\mathrm{H}-\mathrm{CO}_{2}\right]^{-}$, caused by the neutral loss of $\mathrm{CO}_{2}$. Therefore, compound 26 was ascertained as 5,9,12-octadecatrienoic acid [34].

Compound 27 yielded quasi-molecular ion at $m / z 279.2334[\mathrm{M}-\mathrm{H}]^{-}$with elemental composition of $\mathrm{C}_{18} \mathrm{H}_{32} \mathrm{O}_{2}$ (mass error $=$ $-1.65 \mathrm{ppm}$ ). Characteristic ion at $\mathrm{m} / \mathrm{z} 261.2221\left[\mathrm{M}-\mathrm{H}-\mathrm{H}_{2} \mathrm{O}\right]^{-}$was observed after eliminating neutral fragments of $\mathrm{H}_{2} \mathrm{O}$. Hence, compound 27 tentatively identified as (9Z,12Z)-octadeca-9,12-dienoic acid based on mass data.

Compound 28 showed $[\mathrm{M}-\mathrm{H}]^{-}$ion at $\mathrm{m} / \mathrm{z} 255.2327$, and product ions at $\mathrm{m} / \mathrm{z} 237.2202\left[\mathrm{M}-\mathrm{H}-\mathrm{H}_{2} \mathrm{O}\right]^{-}$were obtained by loss of $\mathrm{H}_{2} \mathrm{O}$ in MS/MS spectra. By comparison the literature data[35], compound 28 was tentatively determined as palmitic acid.

\subsubsection{Structural characterization and identification of phenylpropanoids}

Compound 4 exhibited a quasi-molecular ion $\mathrm{m} / \mathrm{z} 417.1406[\mathrm{M}+\mathrm{HCOO}]^{-}$with molecular formula $\mathrm{C}_{17} \mathrm{H}_{24} \mathrm{O}_{9}$. It could generate fragment ions at $m / z 371.1281[\mathrm{M}-\mathrm{H}]^{-}$and $m / z 209.0767\left[\mathrm{M}-\mathrm{H}-\mathrm{C}_{6} \mathrm{H}_{10} \mathrm{O}_{5}\right]^{-}$. According to the reported data [16], compound 4 was characterized as syringin.

Compound 7 produced its precursor ion at $\mathrm{m} / z 677.2288[\mathrm{M}-\mathrm{H}]^{-}$with molecular formula $\mathrm{C}_{29} \mathrm{H}_{42} \mathrm{O}_{18}$ (Fig. 4). The molecular ion generated fragment ions at $m / z 497.1661\left[\mathrm{M}-\mathrm{H}-\mathrm{C}_{6} \mathrm{H}_{12} \mathrm{O}_{6}\right]^{-}$and $m / z 453.1402\left[\mathrm{M}-\mathrm{H}-\mathrm{C}_{6} \mathrm{H}_{12} \mathrm{O}_{6}-\mathrm{CO}_{2}\right]^{-}$, which originated from the neutral loss of $\mathrm{C}_{6} \mathrm{H}_{12} \mathrm{O}_{6}$ and $\mathrm{CO}_{2}$. Comparing with the information of literature [34], compound 7 was tentatively deduced as tangshenoside $\mathrm{I}$.

Figure 4 The MS/MS spectra and the fragmentation pathways of tangshenoside I in negative ion mode. (a) MS/MS spectra;

(b) fragmentation pathways. 
Compounds 8 yielded deprotonated molecular ions at m/z $325.0925[\mathrm{M}-\mathrm{H}]^{-}$, and a glucose residue $\mathrm{C}_{6} \mathrm{H}_{10} \mathrm{O}_{5}$ was eliminated in MS/MS spectrum to form characteristic fragment at $\mathrm{m} / \mathrm{z} 163.0402\left[\mathrm{M}-\mathrm{H}-\mathrm{C}_{6} \mathrm{H}_{10} \mathrm{O}_{5}\right]^{-}$, followed by neutral loss of $\mathrm{CO}_{2}$ presenting $\mathrm{m} / \mathrm{z} 119.0502\left[\mathrm{M}-\mathrm{H}-\mathrm{C}_{6} \mathrm{H}_{10} \mathrm{O}_{5}-\mathrm{CO}_{2}\right]^{-}$. Compound 8 was therefore deduced to be 4-o-beta-glucopyranosyl-ciscoumaric acid combining multi-stage ions information of $\mathrm{MS}^{\mathrm{n}}$ with relevant literatures [16].

Compound 11 generated precursor ion at $\mathrm{m} / z 469.1351[\mathrm{M}-\mathrm{H}]^{-}$with molecular formula $\mathrm{C}_{21} \mathrm{H}_{26} \mathrm{O}_{12}$. The molecular ion was further fragmented into ions at $m / z 325.1046\left[\mathrm{M}-\mathrm{H}-\mathrm{C}_{6} \mathrm{H}_{8} \mathrm{O}_{4}\right]^{-}$and $m / z 163.0393\left[\mathrm{M}-\mathrm{H}_{-} \mathrm{C}_{6} \mathrm{H}_{8} \mathrm{O}_{4}-\mathrm{C}_{6} \mathrm{H}_{10} \mathrm{O}_{5}\right]^{-}$, resulting from the loss of $\mathrm{C}_{6} \mathrm{H}_{8} \mathrm{O}_{4}$, after that the loss of $\mathrm{C}_{6} \mathrm{H}_{10} \mathrm{O}_{5}$. According to the literature data[36], compound 11 was accurately identified as tangshenoside $\mathrm{V}$.

Compound 14 produced $[\mathrm{M}-\mathrm{H}]^{-}$ion at $m / z$ 823.2652, and product ions were further generated at $m / z$ 497.104 1[M-H$\left.\mathrm{C}_{15} \mathrm{H}_{18} \mathrm{O}_{8}\right]^{-}$and $\mathrm{m} / \mathrm{z} 452.9206$ [M-H- $\left.\mathrm{C}_{15} \mathrm{H}_{18} \mathrm{O}_{8}-\mathrm{CO}_{2}\right]^{-}$corresponding to the loss of $\mathrm{C}_{15} \mathrm{H}_{18} \mathrm{O}_{8}$ and $\mathrm{CO}_{2}$. Compound 16 shared an identical elemental composition of $\mathrm{C}_{38} \mathrm{H}_{48} \mathrm{O}_{20}$ as compound 14 , and its fragmentation pathway was in accord with that of compound 4. Codonoside $B$ with higher polarity than Codonoside $A$ was faster eluted on reversed phase chromatography, therefore, compound 14 and compound 16 were distinguished as Codonoside B and Codonoside A [33].

\subsubsection{Structural characterization and identification of hexanol glycosides and hexylene glycosides}

Compounds 5 and 6 were identified primarily as isomers by identical quasi-molecular ions at m/z 423.1865 [M-H] ${ }^{-}$with elemental composition of $\mathrm{C}_{18} \mathrm{H}_{32} \mathrm{O}_{11}$, and their deprotonated molecular ion generated $\left[\mathrm{M}-\mathrm{H}-\mathrm{C}_{6} \mathrm{H}_{10} \mathrm{O}_{5}\right]^{-}$at $\mathrm{m} / \mathrm{z} 261.0986$, resulting from the loss of the $\mathrm{C}_{6} \mathrm{H}_{10} \mathrm{O}_{5}$. They were further differentiated by retention time. Trans-2-hexenyl- $\beta$-glucoside performed stronger polar than cis-2-hexenyl- $\beta$-glucoside, thus was eluted with shorter retention time on reversed phase chromatography. Combining characteristic of the compound and literature data [34], compound 5 and compound 6 were assigned as trans-2-hexenyl- $\beta$-glucoside and cis-2-hexenyl- $\beta$-glucoside.

Compound 9 generated deprotonated ion at $\mathrm{m} / \mathrm{z} 471.2077\left[\mathrm{M}+\mathrm{HCOO}^{-}\right.$, and fragment ions at $\mathrm{m} / z 425.2016[\mathrm{M}-\mathrm{H}]^{-}$was observed by losing $\mathrm{HCOOH}$. In addition, the molecular ion yielded $\left[\mathrm{M}-\mathrm{H}-\mathrm{C}_{6} \mathrm{H}_{10} \mathrm{O}_{5}\right]$ at $\mathrm{m} / z 263.1487$ due to the loss of glucose. Consequently, according to the literature data[16], compound 9 was tentatively identified as hexy- $\beta$-aglucopyranosyl-( $1 \rightarrow 2$ )- $\beta$-a-glucopyranoside. Compound 10 had the same elemental composition of $\mathrm{C}_{18} \mathrm{H}_{34} \mathrm{O}_{11}$ as compound 9, which fragmentation pathway was similar as above. Comparing with the literature [16], compound 10 was tentatively identified as hexy- $\beta$-a-glucopyranosyl-( $1 \rightarrow 6)-\beta$-a-glucopyranoside.

Compound 12 produced a deprotonated ion $m / z 395.1916[\mathrm{M}-\mathrm{H}]^{-}$with molecular formula $\mathrm{C}_{17} \mathrm{H}_{32} \mathrm{O}_{10}$. Product ions at $m / z$ $263.1473\left[\mathrm{M}-\mathrm{H}-\mathrm{C}_{5} \mathrm{H}_{8} \mathrm{O}_{4}\right]^{-}$and $\mathrm{m} / \mathrm{z} 101.0355\left[\mathrm{M}-\mathrm{H}-\mathrm{C}_{5} \mathrm{H}_{8} \mathrm{O}_{4}-\mathrm{C}_{6} \mathrm{H}_{10} \mathrm{O}_{5}\right]^{-}$were generated by continuously losing of pentose and glucose. MS/MS spectra and proposed fragmentation pathway of compound 12 were displayed in Fig. 5, and it was predicatively identified as pentose aldose glucose-n-hexanoside by comparing mass data with the reported data [34].

Figure 5 The MS/MS spectra and the fragmentation pathways of pentose aldose glucose-n-hexanoside in negative ion mode. (a) MS/MS spectra; (b) fragmentation pathways.

\subsubsection{Structural characterization and identification of polyacetylenes}

Compound 13 exhibited its quasi-molecular ion $\mathrm{m} / \mathrm{z} 603.2292[\mathrm{M}+\mathrm{HCOO}]^{-}$with molecular formula $\mathrm{C}_{26} \mathrm{H}_{38} \mathrm{O}_{13}$. It generated a series of ions at $m / z 557.2239[\mathrm{M}-\mathrm{H}]^{-}, m / z 467.1710\left[\mathrm{M}-\mathrm{H}-\mathrm{C}_{7} \mathrm{H}_{6}\right]^{-}$and $m / z 395.1934\left[\mathrm{M}-\mathrm{H}-\mathrm{C}_{6} \mathrm{H}_{10} \mathrm{O}_{5}\right]^{-}$, arising from sequential elimination of $\mathrm{HCOOH}, \mathrm{C}_{7} \mathrm{H}_{6}$ and glucose. Compound 13 was predicatively identified as lobetyolinin based on literature data[16]. 
Compound 15 produced its $[\mathrm{M}+\mathrm{HCOO}]^{-}$ion at $\mathrm{m} / z 441.1780$ with molecular formula $\mathrm{C}_{20} \mathrm{H}_{28} \mathrm{O}_{8}$ (mass error $=-4.48 \mathrm{ppm}$ ). In MS spectrum, it yielded typical ions at $m / z 395.1922[\mathrm{M}-\mathrm{H}]^{-}$and $m / z 215.1083\left[\mathrm{M}-\mathrm{H}-\mathrm{C}_{6} \mathrm{H}_{12} \mathrm{O}_{6}\right]^{-}$, shown in Fig. 6. Therefore, comparing with the literature[33], compound 15 was confirmed as lobetyolin.

Figure 6 The MS/MS spectra and the fragmentation pathways of lobetyolin in negative ion mode. (a) MS/MS spectra; (b) fragmentation pathways.

\subsubsection{Structural characterization and identification of alcohol compound}

Compound 20 exhibited precursor ion at $\mathrm{m} / z 309.2057[\mathrm{M}-\mathrm{H}]^{-}$with molecular formula $\mathrm{C}_{18} \mathrm{H}_{30} \mathrm{O}_{4}$ (Fig. 7), and further generated product ions at $m / z 291.1772\left[\mathrm{M}-\mathrm{H}-\mathrm{H}_{2} \mathrm{O}\right]^{-}$, resulting from the neutral loss of $\mathrm{H}_{2} \mathrm{O}$. According to the related literature[33], compound 20 was unambiguously identified as 6-methylgingediol.

Figure 7 The MS/MS spectra and the fragmentation pathways of 6-methylgingediol in negative ion mode. (a) MS/MS spectra; (b) fragmentation pathways.

\subsubsection{Structural characterization and identification of sesquiterpenes}

Compound 22 yielded the $[\mathrm{M}-\mathrm{H}]^{-}$ion at $\mathrm{m} / \mathrm{z} 247.1352\left(\mathrm{C}_{15} \mathrm{H}_{20} \mathrm{O}_{3}\right.$, mass error $\left.=-2.28 \mathrm{ppm}\right)$. As shown in Fig. 8, fragment ion at $\mathrm{m} / \mathrm{z} 203.1455\left[\mathrm{M}-\mathrm{H}-\mathrm{CO}_{2}\right]^{-}$was obtained in MS/MS spectra. Therefore, comparing with the reported data [16], compound 22 was tentatively identified as atractylenolide III.

Figure 8 The MS/MS spectra and the fragmentation pathways of atractylenolide III in negative ion mode. (a) MS/MS spectra; (b) fragmentation pathways.

\subsection{Method validation}

The calibration curves indicated that the concentration of lobetyolin and atractylenolide III exhibited good linearity with peak areas over the range of $2.08-52.0 \mu \mathrm{g} / \mathrm{ml}$ and $0.236-5.9 \mu \mathrm{g} / \mathrm{ml}$, respectively. The regression equations were $\mathrm{y}=$ $554.29 x+2044.3$ with $R^{2}$ of 0.9990 for lobetyolin, $y=93099 x+14390$ with $R^{2}$ of 0.9994 for atractylenolide III (Fig. S1). Satisfactory results of precision, repeatability and stability were obtained (Table 3). The RSDs within $3 \%$ of precision confirmed good precision. Repeatability of the optimized method was fine with RSDs less than 3\%. Sample of RCR was stable during the tested period with RSDs $₫ 3 \%$. Recovery of lobetyolin was between $95.63 \%$ and $100.07 \%$, while atractylenolide III ranged from 95.66-101.22\%. Entire RSDs within 3\% manifested good recovery.

Table 3

Precision, repeatability and stability of lobetyolin and atractylenolide III $(n=6)$.

\begin{tabular}{|c|c|c|c|c|c|}
\hline \multirow[t]{2}{*}{ Analytes } & \multirow[t]{2}{*}{ Precision (RSD,\%) } & \multirow[t]{2}{*}{ Repeatability (RSD,\%) } & \multirow[t]{2}{*}{ Stability (RSD,\%) } & \multicolumn{2}{|l|}{ Recovery } \\
\hline & & & & Mean(\%) & $\mathrm{RSD}(\%)$ \\
\hline Lobetyolin & 0.57 & 0.87 & 0.51 & 98.21 & 2.15 \\
\hline Atractylenolide III & 1.64 & 2.35 & 2.02 & 98.35 & 2.47 \\
\hline \multicolumn{6}{|c|}{$\begin{array}{l}\text { For } 5 \text {-HMF, a good linearity over the range of } 0.378-7.56 \mu \mathrm{g} / \mathrm{ml} \text { were determined (Fig. S2), and the regression equation } \\
\text { was } y=78068 x-5696.5\left(R^{2}=0.9994\right) . \text { Regarding CPPS, glucose as a reference substance for quantification had } \\
\text { excellent linearity in the range of } 5.015-52.150 \mu \mathrm{g} / \mathrm{mL} \text { (Fig.S3), whose linear return equations was } y=0.0143 x+0.0008 \\
\left(R^{2}=0.9991\right) \text {. }\end{array}$} \\
\hline
\end{tabular}

\subsection{Quantitative results}


The optimized method was performed to estimate dynamic changes of chemical constituents of multiple CR products in processing. In view of sensitivity and specificity of triple quadrupole mass analyzer suitable for accurate quantification, HPLC-QQQ-MS was used to measure contents of lobetyolin and atractylenolide III. Total ion chromatograms for quantifying lobetyolin and atractylenolide III in CR and RCR were shown in Fig. S4. In addition, 5-HMF with strong polar could be eluted fast by HPLC, which was a convenient detection method with relatively low cost. CPPS was well measured by phenolsulphuric acid method. Overall, lobetyolin, atractylenolide III, 5-HMF and CPPS were characteristic components to distinguish between raw $\mathrm{CR}$ and RCR.

The quantitative results of these four active constituents were displayed in Table 4, and their content changing trends were shown in Fig. 9. The content of lobetyolin increased occasionally in the process samples due to sampling errors, but it showed a downward trend as a whole, especially that lobetyolin significantly decreased in RCR compared with crude CR. Similarly, though a rise of atractylenolide III contents in individual samples with processing time, it eventually appeared to be falling after rice-processing. From the fifth minute of the processing, 5-HMF was produced and increased in content gradually. Furthermore, the change of CPPS content showed a overall tendency to reduce during processing.

Table 4

The determination results of compounds in RC and RCR

\begin{tabular}{|lllll|}
\hline Sample & $\begin{array}{l}\text { Lobetyolin } \\
(\mathbf{m g} / \mathbf{g})\end{array}$ & $\begin{array}{l}\text { Atractylenolide III } \\
(\mathbf{m g} / \mathbf{g})\end{array}$ & $\begin{array}{l}\text { 5-HMF } \\
(\mathbf{m g} / \mathbf{g})\end{array}$ & $\begin{array}{l}\text { CPPS } \\
(\%)\end{array}$ \\
\hline CR & 0.2994 & 0.0445 & - & 8.10 \\
\hline CR-1 & 0.3266 & 0.0448 & - & 7.68 \\
\hline CR-2 & 0.3796 & 0.0373 & - & 7.65 \\
\hline CR-3 & 0.2981 & 0.0415 & - & 8.21 \\
\hline CR-4 & 0.3273 & 0.0400 & - & 8.48 \\
\hline CR-5 & 0.3535 & 0.0431 & - & 8.44 \\
\hline CR-6 & 0.3920 & 0.0399 & 0.0326 & 7.48 \\
\hline CR-7 & 0.3073 & 0.0400 & 0.0416 & 6.97 \\
\hline CR-8 & 0.3224 & 0.0369 & 0.0607 & 7.72 \\
\hline CR-9 & 0.2527 & 0.0362 & 0.0748 & 6.54 \\
\hline CR-10 & 0.2807 & 0.0360 & 0.0862 & 5.82 \\
\hline CR-11 & 0.2426 & 0.0350 & 0.0984 & 5.27 \\
\hline RCR & 0.2118 & 0.0384 & 0.1176 & 5.26 \\
\hline
\end{tabular}

Figure 9 The contents of lobetyolin, atractylenolide III, 5-HMF and CPPS in CR and multiple rice-stirring products.

\section{Discussion}

In order to explore changing regularity of components in CR and its rice-processed products, samples used for quantification were took every 1 or 0.5 minutes until processing end time. Then, with a suitable and effective HPLC-MS/MS method for identification and quantification, we demonstrated some changes in chemical composition based on CR stirfried with rice. The reduced content of lobetyolin and atractyllenolide III in RCR indicated some mechanism, namely dehydration and oxidization reaction were involved in the chemical variation [37]. Regarding lobetyolin, several studies had 
shown that it had protective effect in gastric mucosa injury in rats model with gastric ulcer, featuring a better efficacy in low dose than that in high dose [12]. Moreover, certain degree of negative impacts on splenic lymphocyte proliferation and splenic natural killer cell activity were made by lobetyolin [38]. Therefore, the reduction of lobetyolin in RCR might be conducive to better play the role of strengthening the spleen and protecting the stomach. A decline in atractylenolide III content indicated dynamic change during processing, which might be related to thermal instability of and dehydration reaction occurring to form atractylenolide III when the temperature reached a certain degree during heating process [39].

Notably, 5-HMF was newly generated in subsequent rice-processed CR, which implied that Maillard reaction was involved in the processing of stir-frying CR with rice. It was reported that fructose with furan structure was susceptible to Maillard reaction to dehydrate and synthesize 5-HMF [40], leading to speculation that fructose in CR potentially provided the precursor for 5-HMF formation. Maillard reaction products played a vital role in special flavor during materials preparations [41], such as 5-HMF with charred odor, and suitable odors would stimulate endocrine response that affected metabolism [42]. As well, 5-HMF had a certain impact on gastrointestinal movement by promoting excitatory contraction of gastrointestinal smooth muscle [43]. On the one hand, 5-HMF reflected the odor characteristics of RCR; on the other hand, it made contribution to invigorating the spleen and helping with digestion. CPPS was reported as homogeneous polysaccharide, consisting of glucose, fructose, galactose, arabinose, mannose, etc [44]. The content of CPPS was in decline after frying CR with rice, possibly because it was decomposed into oligosaccharides and various reducing sugars, thereby involving in Maillard reaction. Processing principle of RCR for tonifying spleen and dynamic changes of main active ingredients were initially illuminated, whereas it remained further study to prove relevant mechanisms.

\section{Conclusions}

Processing could have influences on content and structural change of the components in traditional Chinese medicine. Herein, a sensitive and rapid approach with UPLC-QTOF-MS/MS was developed for assessing quality of CR and RCR, showing a total of 28 compounds. Moreover, dynamic changes of main chemical components could be found in the processing of stir-frying CR with rice. When CR became to RCR, the contents of lobetyolin, atractylenolide III and CPPS decreased, while that of 5-HMF increased, proving that these four components used for quality control could differentiate the samples before and after processing well. Maillard reaction was potentially formation mechanism of 5-HMF, and the material basis affecting the efficacy of RCR was discussed. Overall, this study has important reference value for investigating the processing chemistry of RCR to some extent.

\section{Abbreviations}

CR: Codonopsis Radix; RCR: rice-processed Codonopsis Radix; 5-HMF: 5-hydroxymethylfurfural; CPPS: codonopsis pilosula polysaccharides; HPLC-MS/MS High-performance liquid chromatography/tandem mass spectrometry; RSD: relative standard deviation.

\section{Declarations}

\section{Acknowledgements}

Not applicable.

\section{Authors' contributions}

QW and YW were responsible for the design of this study. ZL, YW and TL collected and detected samples. QW, DY and YW analyed data. YZ prepared the figures and tables. YZ, YW and CZ writed and revised the article. All authors read and approved the final manuscript. 


\section{Funding}

This work supported by the National Traditional Medicine Standardization Project of China (Grant No. ZYBZH-Y-JIN-34), China Academy of Chinese Medical Sciences (Grant No. ZXKT16001) and project of Beijing for traditional Chinese Medicine Processing Technology inheritance base.

\section{Availability of data and materials}

The datasets used and/or analyzed during the current study are available from the corresponding author on reasonable request.

\section{Ethics approval and consent to participate}

Not applicable.

\section{Consent for publication}

Not applicable.

\section{Competing interests}

All authors declared no competing interests.

\section{Author details}

${ }^{1}$ Institute of Chinese Materia Medica, China Academy of Chinese Medical Sciences, Beijing 100700, China. ${ }^{2}$ College of Pharmacy, Henan University of Chinese Medicine, Zhengzhou 450008, China. ${ }^{3}$ Shanxi Zhendong Genuine Medicinal Materials Development Co., Ltd., Changzhi 047100, China. ${ }^{4}$ Beijing Tongrentang Co., Ltd., Beijing 100062, China.

\section{References}

1. Chinese Pharmacopoeia Commission. Pharmacopeia of the People's Republic of China, Volume 1. Chemical and Industrial Publisher; 2015.

2. Li S, Han Q, Qiao C, et al. Chemical markers for the quality control of herbal medicines: An overview. Chin Med. 2008;3(1):7.

3. Kim EY, Jin AK, Jeon HJ, et al. Chemical fingerprinting of Codonopsis pilosula and simultaneous analysis of its major components by HPLC-UV. Arch Pharmacal Res. 2014;37(9):1148-58.

4. Wang ZT, Ng TB, Yeung HW, et al. Immunomodulatory effect of a polysaccharide-enriched preparation of Codonopsis pilosula roots. Gen Pharmacology the Vascular Syst. 1996;27(8):1347-50.

5. Drug administration of the people's Republic of China. National standard for processing of traditional Chinese Medicine. People's Medical Publishing House; 1988.

6. Hubei Food and Drug Administration. Processing standard of traditional Chinese medicine in Hubei Province. Hubei Science and Technology Press; 2009.

7. Lyu L, Gao J, Li D, et al. Effect of different processing methods on content of indicator components in Codonopsis Radix. J of Shenyang Pharm University. 2020;37(7):650-6.

8. Li P, Qi L, Liu E, et al. Analysis of Chinese herbal medicines with holistic approaches and integrated evaluation models. Trac Trends in Analytical Chemistry. 2008;27(1):66-77.

9. Qing H, Enyuan Z, Zhengtao W, et al. Determination of lobetyolin in Radix Codonopsis by high-performance liquid chromatography. J of Chin Pharm. 2005;40(1):56-8. 
10. Sun Y, Liu J. Structural characterization of a water-soluble polysaccharide from the roots of Codonopsis pilosula and its immunity activity. Int J of Biol Macromol. 2008;43(3):279-82.

11. He J, Ma N, Zhu S, et al. The genus Codonopsis (Campanulaceae): a review of phytochemistry, bioactivity and quality control. J Nat Med. 2015;69(1):1-21.

12. Song D, Wang Z, Li L, et al. Protective effect of lobetyolin on gastric mucosa of experimental gastric ulcer in rats. J of Emerg in Tradit Chin Med. 2008;17(7):963-4.

13. Endo K, Taguchi T, Taguchi F, et al. Antiinflammatory principles of Atractylodes rhizomes. Chem Pharm Bull. 1979;27(12):2954-8.

14. Wang H, Qi J, Han D, et al. Cause and control of Radix Ophiopogonis browning during storage. Chin J Nat Med. 2015;13(1):73-80.

15. Zhou Y, Lei H, Li F, et al. Discussion of processing principle of Dangshen. World Chin Med. 2009;4(3):161-3.

16. Ma X, Leung AKM, Chan CL, et al. UHPLC UHD Q-TOF MS/MS analysis of the impact of sulfur fumigation on the chemical profile of Codonopsis Radix (Dangshen). Analyst. 2014;139(2):505-16.

17. Wu Y, Jiang X, Zhang S, et al. Quantification of flavonol glycosides in Camellia sinensis by MRM mode of UPLC-QQQMS/MS. J Chromatogr B: Anal Technol Biomed Life Sci. 2016;1017-1018:10 - 7.

18. Kumar S, Chandra P, Bajpai V, et al. Rapid qualitative and quantitative analysis of bioactive compounds from Phyllanthus amarus using LC/MS/MS techniques. Ind Crops Prod. 2015;69:143-52.

19. Geng Z, Liu Y, Gou Y, et al. Metabolomics study of cultivated bulbus fritillariae cirrhosae at different growth stages using UHPLC-QTOF-MS coupled with multivariate data analysis. Phytochem Anal. 2018.

20. Wishart DS. Metabolomics: applications to food science and nutrition research. Trends Food Sci Technol. 2008;19(9):482-93.

21. Hummel J, Segu S, Li Y, et al. Ultra performance liquid chromatography and high resolution mass spectrometry for the analysis of plant lipids. Front Plant Sci. 2011;2:54.

22. Li Q, Zhao Y, Zhu D, et al. Lipidomics profiling of goat milk, soymilk and bovine milk by UPLC-Q-Exactive Orbitrap Mass Spectrometry. China Feed. 2017;224:302-9.

23. Wang Q, Jiang P, Ye Fy, et al. Identification and pharmacokinetics of multiple constituents in rat plasma after oral administration of Yinchenzhufu decoction. J Ethnopharmacol. 2014;153(3):714-24.

24. Du Z, Li J, Zhang X, et al. An Integrated LC-MS-Based Strategy for the Quality Assessment and Discrimination of Three Panax Species. Molecules. 2018;23:2988.

25. Nawrot-Hadzik I, Ślusarczyk S, Granica S, et al. Phytochemical diversity in rhizomes of three reynoutria species and their antioxidant activity correlations elucidated by LC-ESI-MS/MS analysis. Molecules. 2019;24:1136.

26. Li Z, Wang Y, Hui O, et al. A novel dereplication strategy for the identification of two new trace compounds in the extract of Gastrodia elata using UHPLC/Q-TOF-MS/MS. J Chromatogr B. 2015;988:45-52.

27. Liu Q, Jiao Z, Liu Y, et al. Chemical profiling of San-Huang decoction by UPLC-ESI-Q-TOF-MS. J Pharm Biomed Anal. 2016;131:20-32.

28. Zhang Y, Guo L, Duan L, et al. Simultaneous determination of 16 phenolic constituents in Spatholobi Caulis by high performance liquid chromatography/electrospray ionization triple quadrupole mass spectrometry. J Pharm Biomed Anal. 2015;102(102):110-8.

29. Chen J, Dia J, Pei W, et al. Tandardization of preparation of rice fried Codonopsis Radix based on correlation analysis of functional component and shape index. China J of Chin materia medica. 2018;43(12):2543-51.

30. Cui B, Xin Y, Ma C. Research progress on the influence of the processing of traditional Chinese medicine on chemical composition and the efficacy. J of Pharm Research. 2019;38(7):403-6. 
31. Bi B. Effect of Processing on the Chemical Components of Traditional Chinese Medicine. Clin J of Tradit Chin Med. 2019;31(5):804-7.

32. Liang Y, Yan G, Wu J, et al. Qualitative and quantitative analysis of lipo-alkaloids and fatty acids in Aconitum carmichaelii using LC-MS and GC-MS. Phytochem Analysis Pca. 2018;29(4).

33. An T, Chen X, Zhang M, et al. Rapid analysis on chemical constituents in roots of Codonopsis tangshen by UPLC coupled with Q-Exactive quadrupole-orbitrap mass spectrometry. Chin Tradit Herb Drug. 2018;49(7):1533-42.

34. Zhang J, Xu X, Huang J, et al. Rapid identification of chemical constituents from Codonopsis Radix by HPLC-LTQOrbitrap-MS ${ }^{n}$. Chin J of Exp Tradit Med Formulae. 2015;21(9):59-63.

35. Wang C, Zhang N, Wang Z, et al. Rapid characterization of chemical constituents of Platycodon grandiflorum and its adulterant Adenophora stricta by UPLC-QTOF-MS/MS. J Mass Spectrom. 2017;52(10):643-56.

36. Zhang J, Xu W, Wang P, et al. Chemical analysis and nulti-component determination in Chinese medicine preparation Bupi Yishen Formula using ultra-high performance liquid chromatography with linear ion trap-Orbitrap mass spectrometry and Triple-Quadrupole tandem mass spectrometry. Front Pharmacol. 2018;9:1-15.

37. Sun X, Wen H, Lu T, et al. Influence of sulfur-fumigation and ventilation baking on contents of atractylenolide I and III in fresh products of atractylodis macrocephalae Rhizoma. Chin J of Exp Tradit Med Formulae. 2016;22(16):10-3.

38. Wang J, Tong X, Li P, et al. Bioactive components on immuno-enhancement effects in the traditional Chinese medicine Shenqi Fuzheng Injection based on relevance analysis between chemical HPLC fingerprints and in vivo biological effects. J Ethnopharmacol. 2014;155(1):405-15.

39. Wang H, Mu S. Problems and countermeasures on primary processing of Atractylodes macrocephala in Enshi. Hans $\mathrm{J}$ of Agricultural Sci. 2017;7(9):655-8.

40. Liu P, Lu X, Li N, et al. Characterization, variables, and antioxidant activity of the Maillard reaction in a fructose(-)histidine model system. Molecules. 2018;24(1):1-15.

41. Nooshkam M, Varidi M, Verma DK. Functional and biological properties of Maillard conjugates and their potential application in medical and food: A review. Food Res Int. 2020;131:109003.

42. Lushchak OV, Carlsson MA, Nassel DR. Food odors trigger an endocrine response that affects food ingestion and metabolism. Cell Mol Life Sci. 2015;72(16):3143-55.

43. Zou L, Qiu B, Liu K, et al. Content of polysaccharide and 5-hydroxymethyl furfural (5-HMF) in Codonopsis Radix after cooked with rice and its impact on rabbit astrointestinal smooth muscle in vitro. Chin Tradit Herb Drug. 2017;48(1):149-54.

44. He L, Zhang J, Liu Z, et al. Isolation, purification and structure identification of Codonopsis pilosula polysaccharide. Chin Tradit Herb Drug. 2008;39(7):986-9.

\section{Figures}



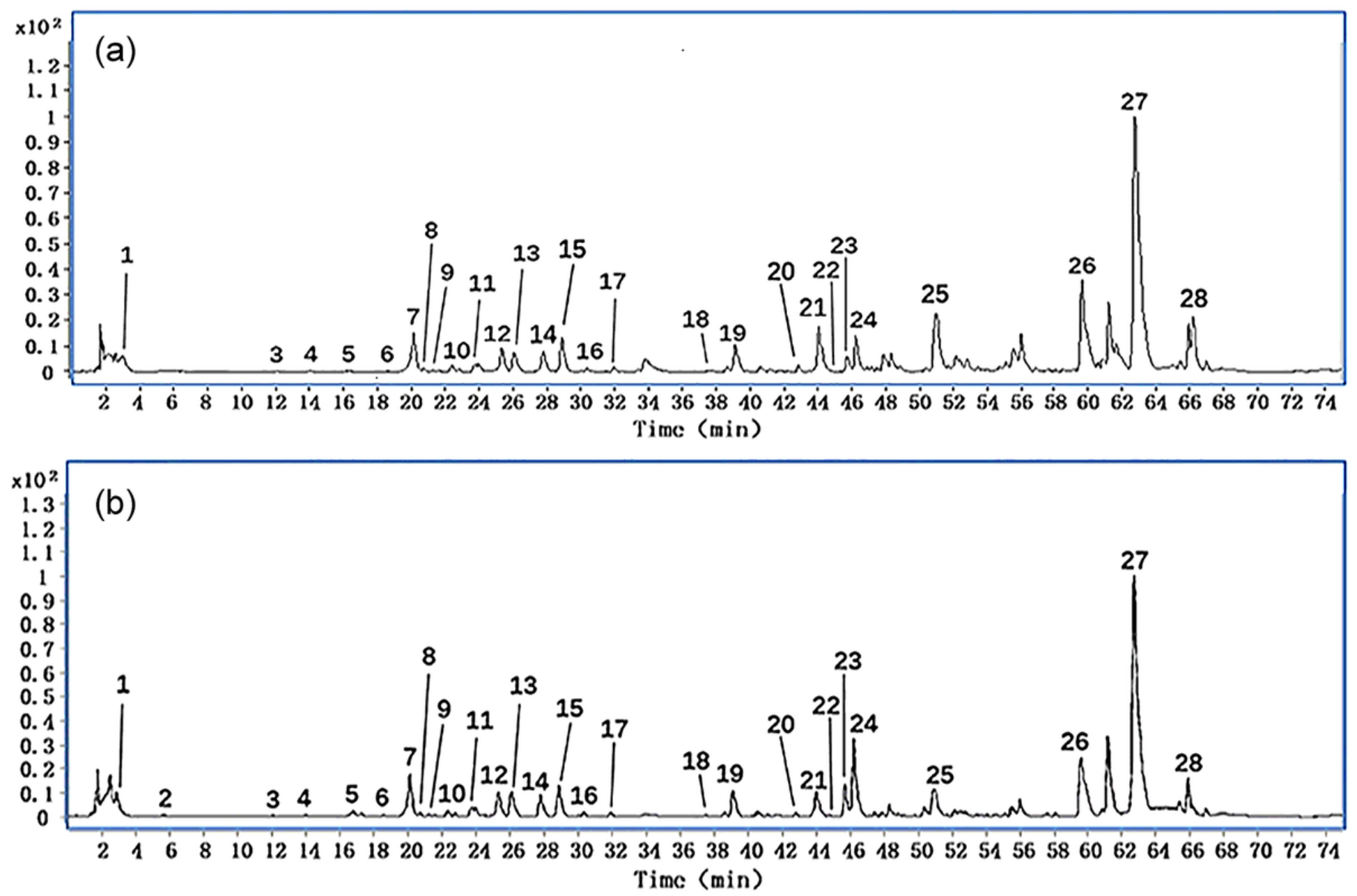

Figure 1

Base peak ion chromatograms of CR (a) and RCR (b) in negative ionization mode.
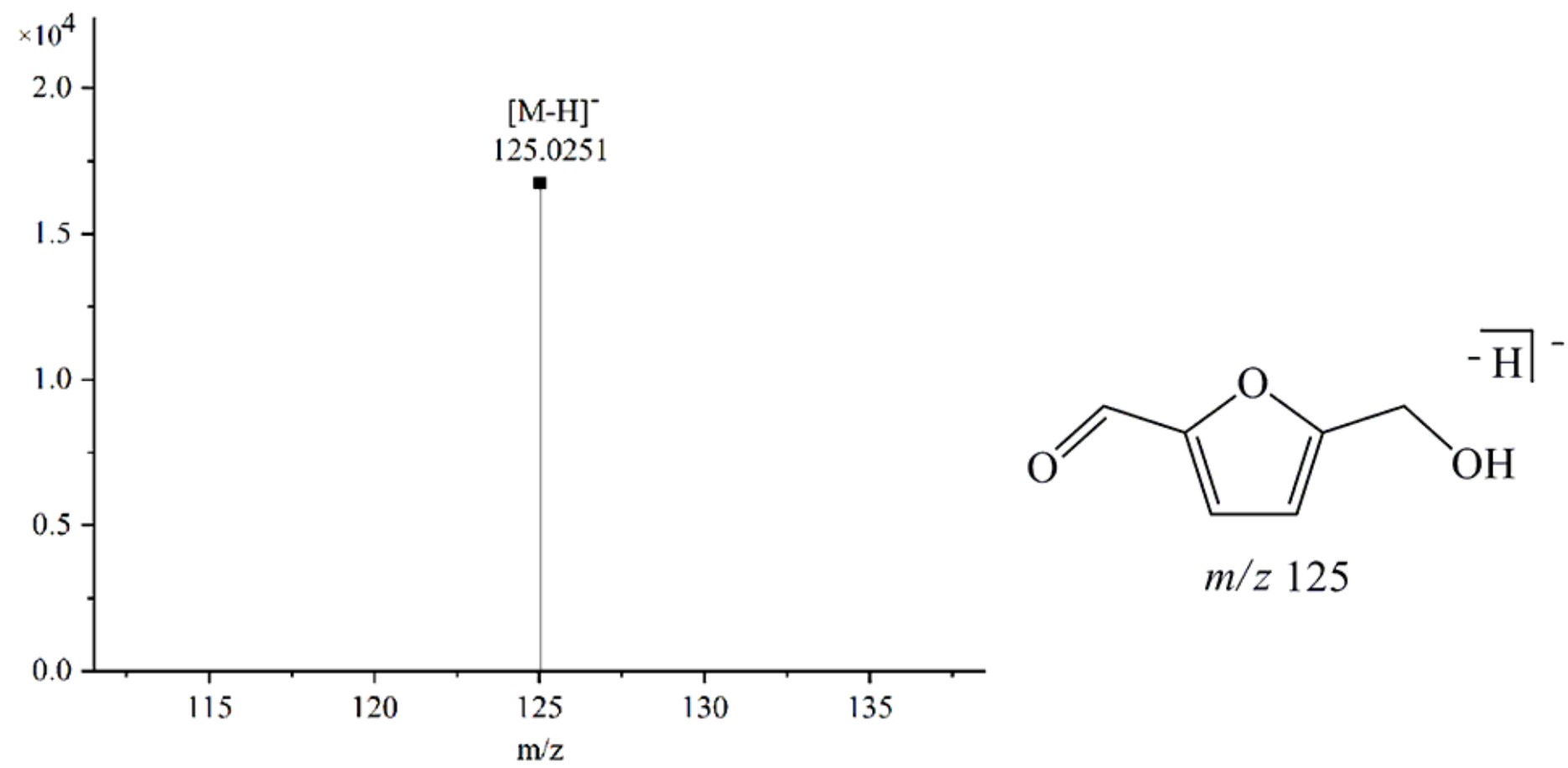

(a)

(b) 


\section{Figure 2}

The mass spectra and the molecular structure of 5-HMF in negative ion mode. (a) mass spectra; (b) molecular structure.

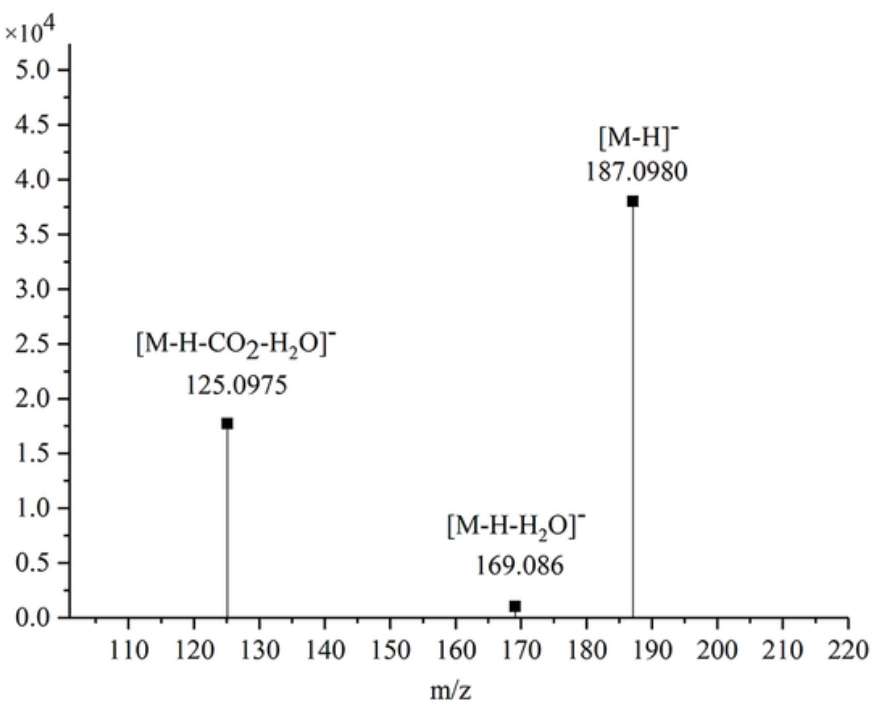

(a)

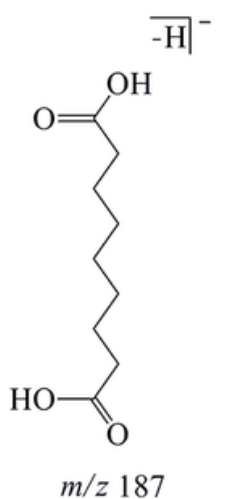

(b)

\section{Figure 3}

The MS/MS spectra and the fragmentation pathways of azelaic acid in negative ion mode. (a) MS/MS spectra; (b) fragmentation pathways.

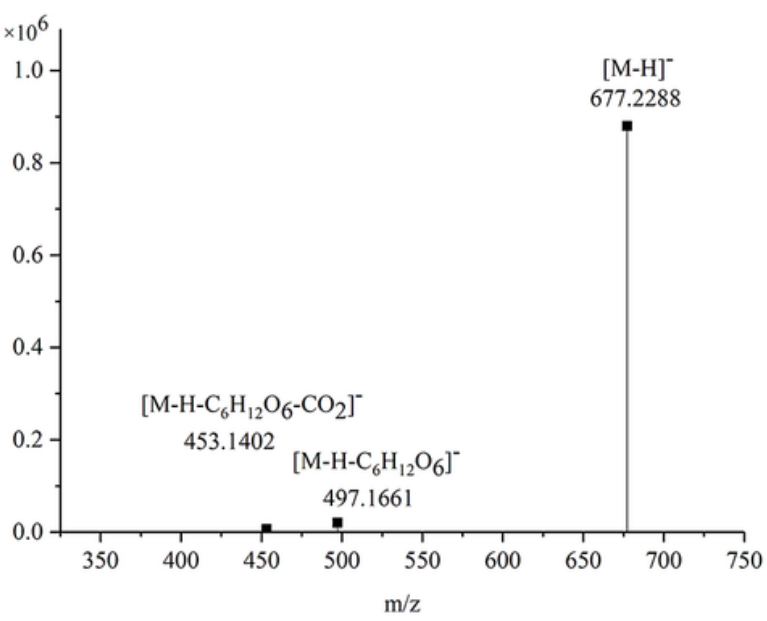

(a)

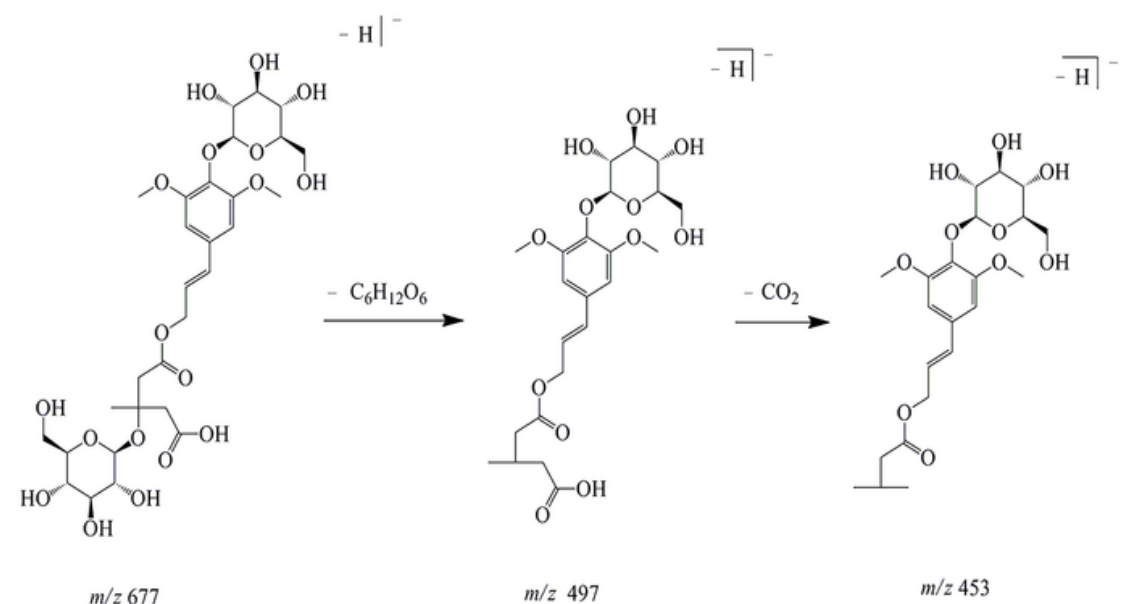

(b)

\section{Figure 4}

The MS/MS spectra and the fragmentation pathways of tangshenoside I in negative ion mode. (a) MS/MS spectra; (b) fragmentation pathways. 


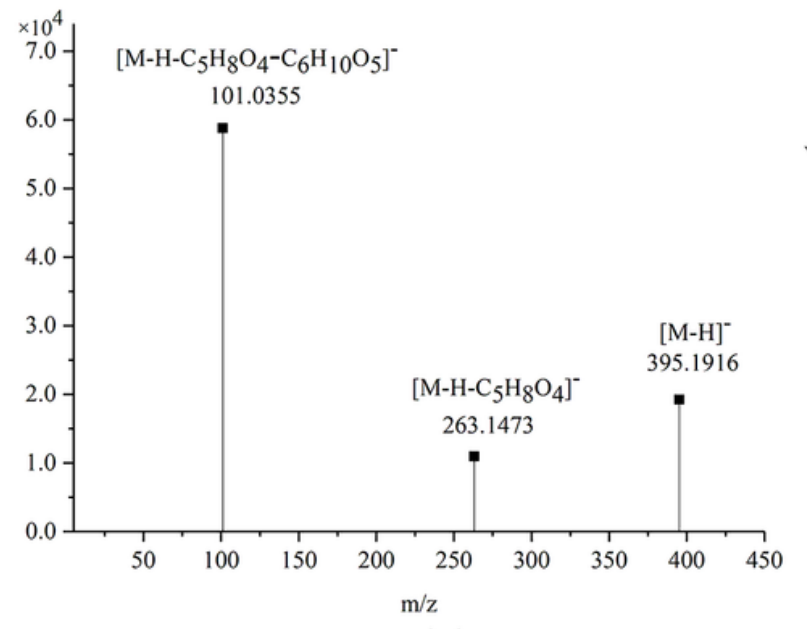

(a)

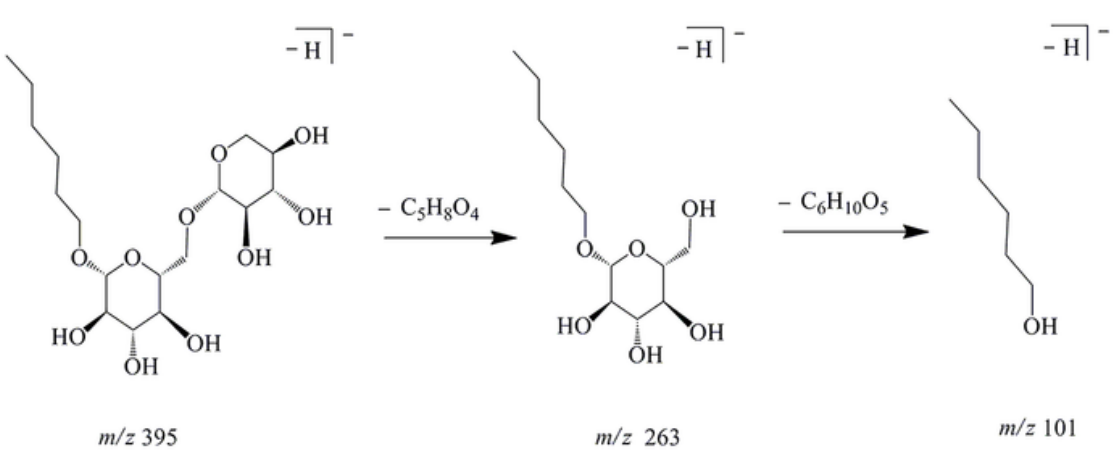

(b)

\section{Figure 5}

The MS/MS spectra and the fragmentation pathways of pentose aldose glucose-n-hexanoside in negative ion mode. (a) MS/MS spectra; (b) fragmentation pathways.

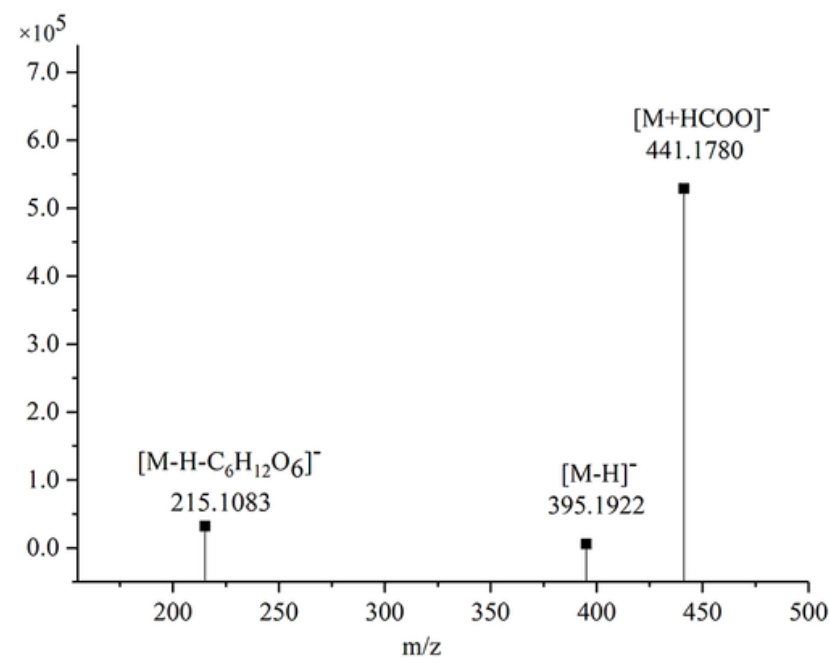

(a)
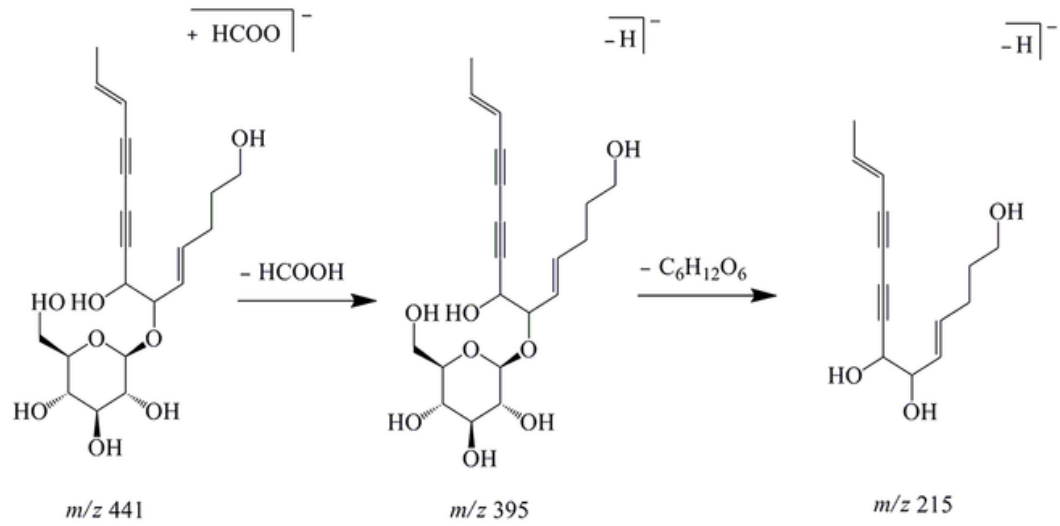

$m / z 215$

Figure 6

The MS/MS spectra and the fragmentation pathways of lobetyolin in negative ion mode. (a) MS/MS spectra; (b) fragmentation pathways. 


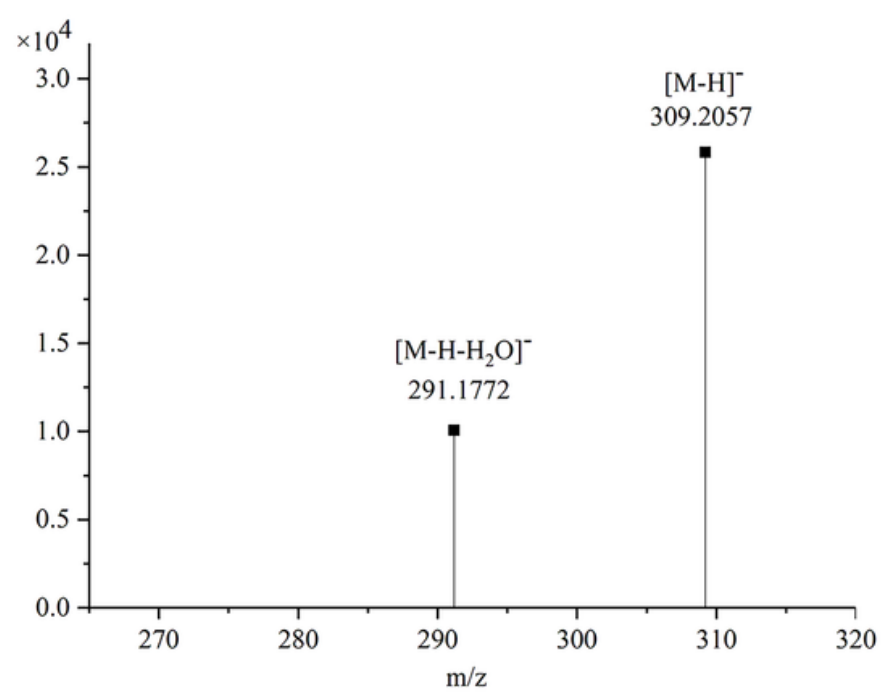

(a)
$-\mathrm{H}^{-}$

$-\left.\mathrm{H}\right|^{-}$

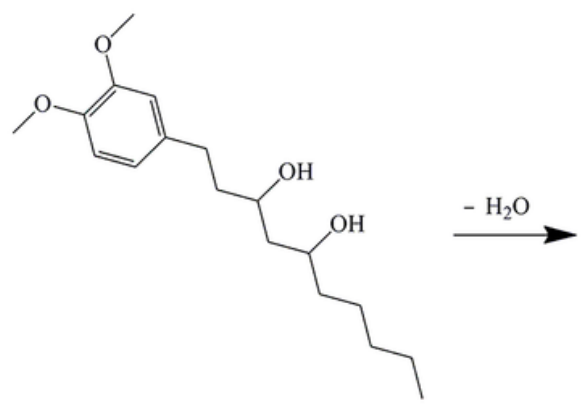

$m / z 309$

(b)

\section{Figure 7}

The MS/MS spectra and the fragmentation pathways of 6-methylgingediol in negative ion mode. (a) MS/MS spectra; (b) fragmentation pathways.

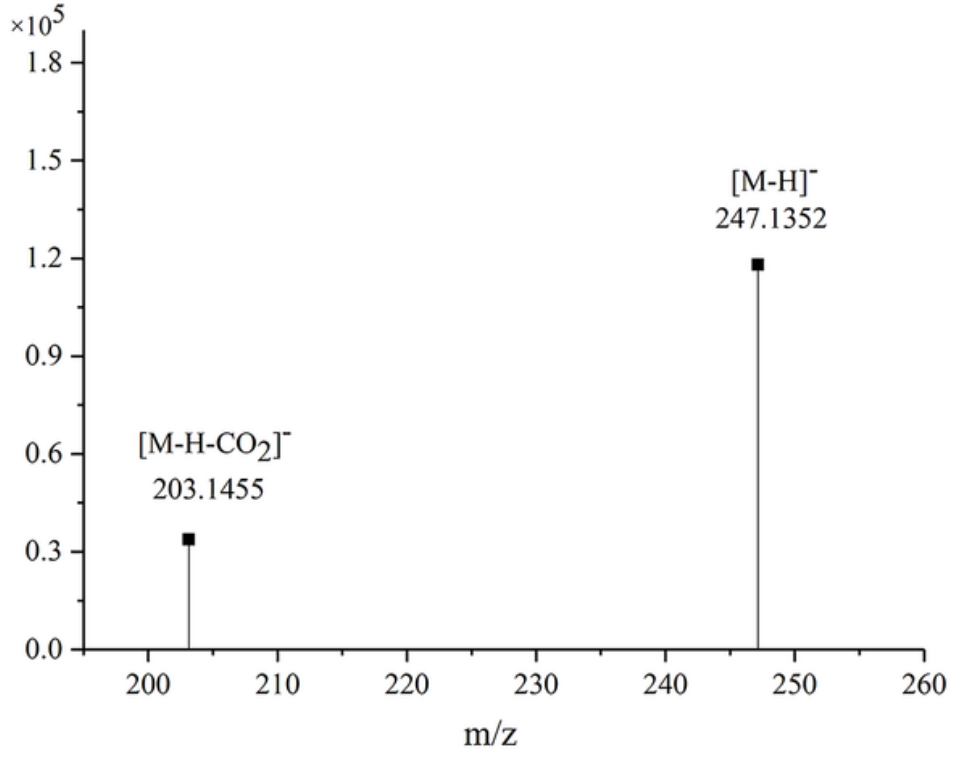

(a)

$-\left.\mathrm{H}\right|^{-}$

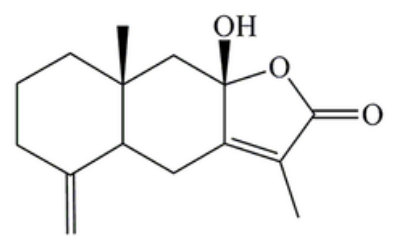

$\mathrm{m} / \mathrm{z} 247$

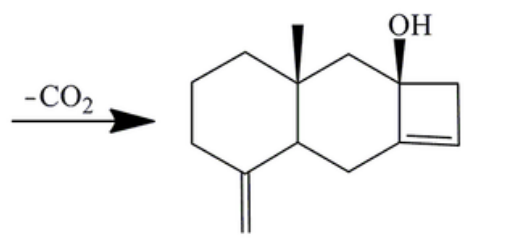

$m / z 203$

(b)

\section{Figure 8}

The MS/MS spectra and the fragmentation pathways of atractylenolide III in negative ion mode. (a) MS/MS spectra; (b) fragmentation pathways. 

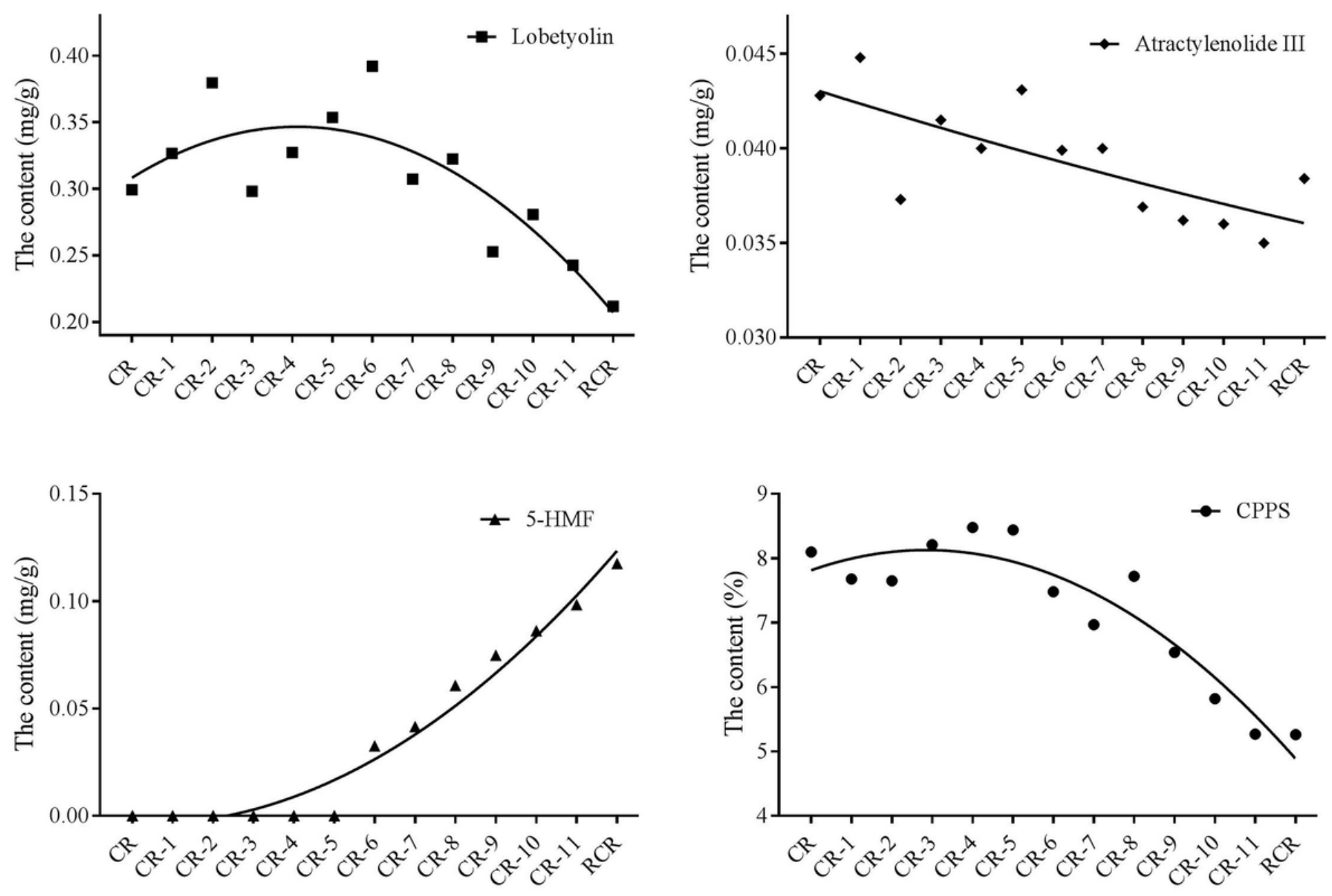

Figure 9

The contents of lobetyolin, atractylenolide III, 5-HMF and CPPS in CR and multiple rice-stirring products.

\section{Supplementary Files}

This is a list of supplementary files associated with this preprint. Click to download.

- SupplementaryMaterial.docx 